\title{
SNMMI/ASNC/SCCT Guideline for Cardiac SPECT/CT and PET/CT 1.0*
}

Sharmila Dorbala ${ }^{1}$, Marcelo F. Di Carli ${ }^{1}$, Dominique Delbeke ${ }^{2}$, Suhny Abbara ${ }^{3}$, E. Gordon DePuey ${ }^{4}$, Vasken Dilsizian ${ }^{5}$, Joey Forrester ${ }^{2}$, Warren Janowitz ${ }^{6}$, Philipp A. Kaufmann ${ }^{7}$, John Mahmarian ${ }^{8}$, Stephen C. Moore ${ }^{1}$, Michael G. Stabin ${ }^{2}$, and Paul Shreve ${ }^{9}$

${ }^{1}$ Brigham and Women's Hospital, Boston, Massachusetts; ${ }^{2}$ Vanderbilt University Medical Center, Nashville, Tennessee; ${ }^{3}$ Massachusetts General Hospital, Boston, Massachusetts; ${ }^{4}$ St. Luke's-Roosevelt Hospital, New York, New York; ${ }^{5}$ University of Maryland Medical Center, Baltimore, Maryland; ${ }^{6}$ Baptist Hospital of Miami, Miami, Florida; ${ }^{7}$ University Hospital Zurich, Zurich, Switzerland; ${ }^{8}$ Methodist Hospital, Houston, Texas; and ${ }^{9}$ Advanced Radiology Services, Grand Rapids, Michigan

\section{INDEX}

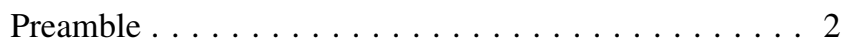

I. Introduction $\ldots \ldots \ldots \ldots \ldots \ldots$

II. Goals . . . . . . . . . . . . . . . . . . 2

III. Definitions ................ 3

IV. Common clinical applications . . . . . . . . 3

V. Qualifications and responsibilities of personnel (in the United States) ............... 3

A. Physicians ............... 3

1. SPECT/CT cardiac radionuclide imaging . . . . . . . . . . . . 3

2. PET/CT cardiac radionuclide imaging. . . 3

3. Cardiovascular stress testing .......4 4

4. Cardiac CT ............. . 4

B. Technologists . . . . . . . . . . . . . 4

1. SPECT/CT cardiac radionuclide imaging . . . . . . . . . . 4

2. PET/CT cardiac radionuclide imaging. . . 4

3. Cardiac CT .............. 4

C. Physicists. . . . . . . . . . . . . 5

D. The institution and equipment ....... 5

VI. Procedure/specifications of the examination .....6 6

A. Patient screening. ............6 6

B. Patient preparation and precautions .....6 6

1. NPO ...............6 6

2. Exercise. .............. 6

3. Caffeine. .............. 6

4. Contrast allergy ............66

5. Intravenous access ........... 6

6. Breathing instructions .........6 6

7. Medications ...............6 6

Received Feb. 27, 2012; revision accepted Feb. 27, 2012.

For correspondence or reprints contact: Sharmila Dorbala, Brigham and Women's Hospital, 70 Francis St., Boston, MA 02115.

E-mail: sdorbala@partners.org

*NOTE: YOU CAN ACCESS THIS GUIDELINE THROUGH THE SNMMI

WEB SITE (http://www.snmmi.org/guidelines).

Published online Jun. 18, 2013.

COPYRIGHT ( 2013 by the Society of Nuclear Medicine and Molecular Imaging, Inc.

DOI: 10.2967/jnumed.112.105155
8. Electrocardiogram (ECG) leads . . . . . . 7

9. Position . . . . . . . . . . . . . . 7

10. Breast feeding ........... 7

C. Information pertinent to the procedure. . . . 7

1. History . . . . . . . . . . . 7

2. Scan time .............. 7

3. Breath-hold .............. . . 7

4. Informed consent . . . . . . . . . . 7

D. Radiopharmaceuticals . . . . . . . . . . . 7

E. Acquisition protocols . . . . . . . . . . . 7

1. SPECT/CT MPI . . . . . . . . . . 7

2. PET/CT MPI . . . . . . . . . . 7

3. ${ }^{18}$ F-FDG PET/CT . . . . . . . . . . 8

4. Attenuation-correction CT scan (CTAC) . . . . . . . . . . . 10

5. Coronary artery calcium scan. ..... 11

6. Coronary CTA . . . . . . . . . . . . . 11

7. Fusion of radionuclide and CT images . . . 13

F. Integrating diagnostic information from radionuclide and CT images . . . . . . . . 13

1. Coronary artery calcium scoring and MPI ................. 13

2. Coronary CTA and MPI ......... 13

3. Indications and appropriate use criteria for hybrid imaging. . . . . . . . 13

4. Fusion of radionuclide and CT images . . . 14

5. CTAC and emission images . . . . . . . . 14

6. CTA and emission images . . . . . . . 14

VII. Documentation/reporting . . . . . . . . . . . . 14

VIII. Equipment specification . . . . . . . . . . . 16

IX. Quality control (QC) and improvement, safety,

infection control, and patient education

concerns. . . . . . . . . . . . . 16

A. Hardware QC . . . . . . . . . . . . 16

B. Imaging QC . . . . . . . . . . 16

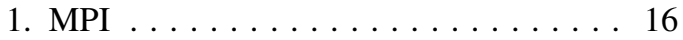

2. CT ............. 16

3. Imaging QC unique to hybrid devices .... 16

4. Fusion software ............ 16

5. Sources of error ............ 17 
X. Radiation safety and ALARA issues....... 19

A. MPI dosimetry ............. 19

B. MPI in the pregnant or potentially pregnant

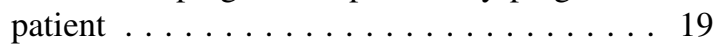

C. MPI in the breast-feeding patient. ...... 19

D. Coronary CTA. . . . . . . . . . . 20

XI. Conclusion. . . . . . . . . . . . . . . . 20

XII. References................... . 20

XIII. Approval . . . . . . . . . . . . . . . 23

\section{PREAMBLE}

The Society of Nuclear Medicine and Molecular Imaging (SNMMI) is an international scientific and professional organization founded in 1954 to promote the science, technology, and practical application of nuclear medicine. Its 16,000 members are physicians, technologists, and scientists specializing in the research and practice of nuclear medicine. In addition to publishing journals, newsletters, and books, the SNMMI also sponsors international meetings and workshops designed to increase the competencies of nuclear medicine practitioners and to promote new advances in the science of nuclear medicine.

The SNMMI will periodically define new guidelines for nuclear medicine practice to help advance the science of nuclear medicine and to improve the quality of service to patients throughout the United States. Existing practice guidelines will be reviewed for revision or renewal, as appropriate, on their fifth anniversary or sooner, if indicated.

Each practice guideline, representing a policy statement by the SNMMI, has undergone a thorough consensus process in which it has been subjected to extensive review, requiring the approval of the Committee on Guidelines and SNMMI Board of Directors. The SNMMI recognizes that the safe and effective use of diagnostic nuclear medicine imaging requires specific training, skills, and techniques, as described in each document. Reproduction or modification of the published practice guideline by those entities not providing these services is not authorized.

These guidelines are an educational tool designed to assist practitioners in providing appropriate care for patients. They are not inflexible rules or requirements of practice and are not intended, nor should they be used, to establish a legal standard of care. For these reasons and those set forth below, the SNMMI cautions against the use of these guidelines in litigation in which the clinical decisions of a practitioner are called into question.

The ultimate judgment regarding the propriety of any specific procedure or course of action must be made by the physician or medical physicist in light of all the circumstances presented. Thus, there is no implication that an approach differing from the guidelines, standing alone, was below the standard of care. To the contrary, a conscientious practitioner may responsibly adopt a course of action different from that set forth in the guidelines when, in the reasonable judgment of the practitioner, such course of action is indicated by the condition of the patient, limitations of available resources, or advances in knowledge or technology subsequent to publication of the guidelines.

The practice of medicine involves not only the science, but also the art, of dealing with the prevention, diagnosis, alleviation, and treatment of disease. The variety and complexity of human conditions make it impossible to always reach the most appropriate diagnosis or to predict with certainty a particular response to treatment. Therefore, it should be recognized that adherence to these guidelines will not ensure an accurate diagnosis or a successful outcome. All that should be expected is that the practitioner will follow a reasonable course of action based on current knowledge, available resources, and the needs of the patient to deliver effective and safe medical care. The sole purpose of these guidelines is to assist practitioners in achieving this objective.

\section{INTRODUCTION}

This guideline was developed collaboratively by the SNMMI with the American Society of Nuclear Cardiology (ASNC) and the Society of Cardiovascular CT (SCCT).

Since 2002, there has been a rapid evolution of hybrid imaging technology incorporating high-quality multidetectorrow CT technology, along with the latest SPECT and PET detector systems. The increasing clinical use of these hybrid systems for cardiovascular radionuclide studies necessitates the establishment of guidelines to ensure reliable use and practice of this technology. The purpose of this document is to assist physicians in the proper performance (acquisition, processing, interpretation, and reporting) of cardiovascular imaging procedures using hybrid-imaging devices and in the use of postprocessing software techniques to fuse cardiovascular images obtained on dedicated CT and radionuclide scanners. This document assumes a basic knowledge of cardiac SPECT (1$5)$ and PET $(1,4,6)$. For further details, the readers are referred to the previously published guideline documents. The current document will focus on the use of hybrid imaging procedures and cardiac CT.

\section{GOALS}

Hybrid imaging is the term frequently used to describe imaging approaches that combine radionuclide imaging with CT. This could be achieved with both $\mathrm{CT}$ and radionuclide scanners mounted on the same gantry or with separate imaging systems. The radionuclide component of hybrid imaging could include either SPECT or PET. The CT component of the original hybrid systems was designed primarily for attenuation correction and not for diagnostic anatomic imaging. However, the more recent SPECT/CT and PET/CT scanners incorporate multidetector CT scanners that provide diagnostic-quality CT, including coronary artery calcium scoring and coronary CT angiography (CTA).

Hybrid SPECT/CT and PET/CT enable us to assess cardiac physiology along with cardiac and coronary anatomy (7-9). The use of CT-based transmission imaging and attenuation correction improves the diagnostic accuracy of myocardial perfusion imaging (MPI), compared with non-attenuation-corrected MPI (10). Attenuation correction facilitates quantitative imaging of myocardial perfusion and metabolism. The assessment of anatomic coronary atherosclerotic burden may amplify the diagnostic and prognostic value of rest and stress MPI. Lastly, the fusion of cardiac and coronary anatomy with functional imaging holds potential for research applications in molecular imaging. Data to support the clinical utility of hybrid imaging continue to accrue (11). This document provides a consensus recommendation for how best to perform, interpret, and report clinical hybrid cardiac imaging studies. 


\section{DEFINITIONS}

A hybrid SPECT/CT scanner is an integrated device wherein the gantry includes both a SPECT scanner and a CT scanner, which use a common bed to move the patient sequentially through both scanners. A hybrid PET/CT scanner is an integrated device wherein the gantry includes both a PET scanner and a CT scanner, also using a common bed to move the patient sequentially through both scanners. A dedicated SPECT scanner is a SPECT scanner with or without a radionuclide source for transmission imaging and attenuation correction. A dedicated PET scanner is one containing a radionuclide source for transmission imaging and attenuation correction. CTAC refers to a CT transmission scan used for attenuation correction of the radionuclide images. Misregistration refers to the misalignment of SPECT or PET emission images with their corresponding CT scans. SPECT/CT or PET/CT image fusion refers to the process of registration and display of the radionuclide and CT images. The term MPI is used in this document to refer to MPI with either SPECT or PET unless otherwise specified.

\section{COMMON CLINICAL APPLICATIONS}

The clinical applications of hybrid cardiac imaging include but are not limited to attenuation-corrected MPI, coronary artery calcium scoring, coronary CTA, fusion imaging and localization, cardiac viability, and imaging of inflammatory cardiac conditions.

Attenuation correction is routinely performed for MPI studies on hybrid devices and on some dedicated scanners, whereas coronary artery calcium scoring and coronary CTA are performed for specific clinical indications. Appropriate use criteria for cardiovascular CT (12) and for radionuclide cardiac imaging (4) have been addressed by the American College of Cardiology and the American College of Radiology, in collaboration with other organizations, including the SNMMI.

See also part F of section VI, "Integrating Diagnostic Information from Radionuclide and CT Images," and supporting references.

\section{QUALIFICATIONS AND RESPONSIBILITIES OF PERSONNEL (IN THE UNITED STATES)}

\section{A. Physicians}

Presently, there is no specific credentialing or qualification requirement for the performance and interpretation of hybrid imaging. Physicians with documented training in PET or SPECT and cardiac CT are generally qualified to perform and interpret hybrid-imaging procedures. These physicians should be listed as an authorized user on the radioactive materials license of their institution. When required by the Nuclear Regulatory Commission or by the state, at least one physician member of the facility must be a participating member of the committee that deals with radiation safety. Physicians trained in nuclear cardiology, and nuclear medicine physicians who are not radiologists, will require additional training in $\mathrm{CT}$ detector physics and instrumentation, as well as training in performing and interpreting cardiac CT (13), except when solely interpreting imaging from scanners with extremely low-dose CT and interpretable CT images (XACT [Digirad], Brightview XCT [Philips], and possibly Hawkeye [GE Healthcare]). Physicians trained primarily in $\mathrm{CT}$ will require additional time to learn the physics and instrumentation of radionuclide imaging, as well as training to perform and interpret radionuclide images and to understand radionuclide physiology, radionuclide handling, and radiation safety for patients and personnel (14). Physicians supervising and performing stress testing must be experienced in exercise and pharmacologic stress testing with vasodilator agents and dobutamine (15). In addition to being a qualified physician with a license to practice medicine, physicians should have the specific qualifications listed below for performing and interpreting hybrid imaging.

1. SPECT/CT cardiac radionuclide imaging

The credentialing requirements for physicians to perform and interpret SPECT and SPECT/CT cardiac radionuclide imaging include training and certification. There are 3 groups of professional organizations that have recommendations for training in cardiac SPECT, PET, and/ or CT: nuclear medicine, radiology, and cardiology. SNMMI recommends that all nuclear medicine examinations should be performed under the supervision of, and interpreted by, a physician certified in nuclear medicine or nuclear radiology by the American Board of Nuclear Medicine, the American Board of Radiology, the Royal College of Physicians or Surgeons of Canada, Le College des Medecins du Quebec, or the equivalent (16). ASNC endorsed the training in nuclear cardiology published by the American College of Cardiology Foundation (ACCF). Level 1 (2 mo) training makes the trainee conversant with the field of nuclear cardiology for application in general clinical management of cardiovascular patients. Level 2 (4 mo) training provides the trainee with special expertise to practice clinical nuclear cardiology. Level 2 training requires a minimum of 4 mo of training or experience in cardiovascular nuclear medicine, with interpretation of 300 cases under the supervision of a qualified physician. For 35 of these cases (25 MPI and 10 radionuclide angiocardiography/gated blood pool imaging/ventriculography), the physician being trained must be present and involved in the acquisition of the studies. A minimum of 30 cases with catheter or CT coronary angiographic correlation must be documented (17). Alternatively, training can meet the requirements of the American College of Radiology (ACR)/SNMMI Technical Standard for Diagnostic Procedures Using Radiopharmaceuticals (18).

Certification can be by any of the following specialty boards or certifying bodies: the American Board of Nuclear Medicine; the American Board of Radiology; the American Board of Radiology with subspecialty certification in nuclear radiology; the American Board of Internal Medicine, including certification in cardiovascular disease; and the Certification Board of Nuclear Cardiology.

2. PET/CT cardiac radionuclide imaging

The credentialing requirements for physicians for PET/CT cardiac radionuclide imaging include meeting the qualifications stated above for SPECT and SPECT/ CT cardiac radionuclide imaging, with additional specific experience in cardiac PET studies.

A minimum of 4 mo of training or experience is required in cardiovascular nuclear medicine, with interpretation of 300 cases under the supervision of a qualified 
physician. For 35 of these cases, the physician being trained must be present and involved in the acquisition of the studies, which must include a reasonable distribution of cardiac PET studies (14). Evidence of participation and maintenance of certification is as required by the specialty board (14). Demonstration of continuing competence is required through interpretation and reporting of at least 50 cardiac PET/CT examinations per year (14).

3. Cardiovascular stress testing

The physician supervising the stress portion of the study and interpreting stress radionuclide cardiac imaging must acquire the necessary cognitive skills as defined by the clinical competence statement on stress testing by the American College of Cardiology/American Heart Association (15). The skills necessary for supervision or interpretation of stress testing can be acquired through multiple pathways, including residency training in nuclear medicine (19), a fellowship in cardiovascular disease, or electives during internal medicine or family practice residency (15). Trainees should devote at least $4 \mathrm{wk}$ to this training and should participate in at least 50 procedures to achieve competence in supervision and interpretation (15). For physicians who did not receive formal training for the supervision or interpretation of stress testing during a residency or fellowship but who have performed stress testing on a regular and substantial basis for more than 3 y (minimum, 150 procedures), experience may be considered individually in lieu of formal training and should meet the criteria as described by the clinical competence statement on stress testing (15).

It is recommended that physicians perform at least 25 exercise tests per year to maintain their competence, with a quality assurance program and successful completion of a course in advanced cardiovascular life support that is renewed on a regular basis (15).

4. Cardiac CT

Physicians must have substantial knowledge of radiation biology; the physics of CT; the principles of CT image acquisition and postprocessing; the design of CT protocols, including the rate and timing of contrast administration; and education and competency in all aspects of cardiac imaging such as cardiac anatomy, physiology, pathology, or cardiac CT $(13,20)$. Physicians involved in coronary CTA must be familiar with the potential hazards associated with $\mathrm{CT}$ and the intravenous administration of iodinated contrast media $(13,20)$.

For cardiologists, the minimum requirement to independently perform and interpret cardiac CT is level 2 training $(20,21)$. This includes interpretation of at least 50 unenhanced and 150 contrast-enhanced studies (in which the physician is physically present and involved in image acquisition and interpretation for $35 \mathrm{CT}$ studies if a fellow in training and 50 cases if a physician in practice) and completion of didactic studies dedicated to cardiac CT $(21,22)$. Radiologists should meet the requirements of the ACR Practice Guideline for the Performance and Interpretation of Cardiac CT. Nonradiologist nuclear medicine physicians should meet either the requirements for cardiologists or the requirements for radiologists.
Physicians will be responsible for all aspects of hybrid imaging, including reviewing the clinical indications for the study, specifying the study protocol and the methods of image reconstruction, interpreting the images, and generating a report (13). The physician is responsible for the completeness of the interpretation of coronary, cardiac, and noncardiac findings (normal or abnormal) observed during the examination.

Physicians should interpret a sufficient number of studies to maintain competence, participate in CME activities relevant to their practice, and participate in a quality program to assess interpretation accuracy.

\section{B. Technologists}

Nuclear medicine technologists are board-certified by the Nuclear Medicine Technology Certification Board or the American Registry of Radiologic Technologists to perform conventional imaging procedures in nuclear medicine and PET. The credentialing requirements for nuclear medicine technologists to perform hybrid imaging vary from state to state and may be regulated by the Nuclear Regulatory Commission. There is a growing emphasis on credentialing in CT for technologists involved in SPECT/CT or PET/CT. The Society of Nuclear Medicine and Molecular Imaging Technologist Section completed a document in 2008 detailing the scope of practice for the nuclear medicine technologist, which includes transmission scanning with $\mathrm{CT}$ in hybrid systems (SPECT/CT and PET/CT (23)). The present requirements in general are as follows:

1. SPECT/CT cardiac radionuclide imaging

A registered nuclear medicine technologist with the credentials RT (N) or CNMT (Certified Nuclear Medicine Technologist) may operate the SPECT scanner. For SPECT/CT, technologists involved must be well educated and trained in SPECT/CT procedures and radiation protection principles (24). Depending on state and federal regulations, a technologist with one of these credentials may operate hybrid equipment after obtaining appropriate additional education and training and demonstrating competency. Nuclear medicine technologists can obtain additional credentialing in nuclear cardiology through a specialty examination offered by the Nuclear Medicine Technology Certification Board.

2. PET/CT cardiac radionuclide imaging

A registered nuclear medicine technologist with the credentials RT $(\mathrm{N})$ or CNMT may operate the PET scanner. For PET/CT, technologists involved must be well educated and trained in PET/CT procedures and radiation protection principles (25). Depending on state and federal regulations, a technologist with one of these credentials may operate hybrid equipment after obtaining appropriate additional education and training and demonstrating competency. Nuclear medicine technologists can obtain additional credentialing in PET through a specialty examination offered by the $\mathrm{Nu}$ clear Medicine Technology Certification Board.

3. Cardiac CT

A technologist for cardiac CT must be a registered radiographer with the credentials RT (T), RT (R), experienced at operating the $\mathrm{CT}$ scanner and with experience in cardiac imaging (13). 
There are at least 2 technologist issues related to hybrid SPECT/CT and PET/CT (Table 1). The first is training of the nuclear medicine technologists on these hybrid scanners. The next is education of the technologists in cardiac physiology and anatomy. Training related to the technical aspects of radionuclide imaging on the hybrid devices, CT, and processing for attenuation correction, calcium scoring, and coronary CTA studies is necessary. Since hybrid imaging devices capture exquisite anatomic detail of the heart and chest, compared with radionuclide imaging, cardiovascular anatomy and physiology training is also required. For example, the technologist would need to identify the anatomic landmarks of the carina and the heart borders on the CT scan to position patients for emission scans. Identification of the origin of the left main coronary artery is important for positioning the start and end locations for coronary CTA studies. Therefore, with the abovementioned training and an additional credential in CT through a specialty examination offered by the American Registry of Radiologic Technologists, nuclear medicine technologists are qualified to perform cardiac CT on hybrid scanners provided the state licensing boards recognize this credential (26).

\section{Physicists}

A medical physicist for SPECT/CT, PET/CT, and CT must show competence to practice independently in one or more of the subcategories in medical physics through previous experience or education (13). The appropriate subfields for medical physics include therapeutic radiologic, medical nuclear, and radiologic physics (13).

The recommended certification for CT is that by the American Board of Radiology, in Radiologic Physics or Diagnostic Radiology Physics; the American Board of Medical Physics, in Diagnostic Imaging Physics; or the Canadian College of Physicists in Medicine, in Diagnostic Radiologic Physics (13).
The recommended certification for nuclear medicine and PET is that by the American Board of Radiology, in Radiologic Physics or Medical Nuclear Physics; the American Board of Medical Physics, in Nuclear Medicine Physics; the Canadian College of Physicists in Medicine, in Nuclear Medicine Physics; or the American Board of Science in Nuclear Medicine, in Nuclear Medicine Physics and Instrumentation.

\section{The institution and equipment}

Per the Medicare Improvements for Patients and Providers Act of 2008 (27), outpatient imaging facilities (not hospitals) should meet the standards of accreditation set forth by the applicable societies, Intersocietal Commission for the Accreditation of Nuclear Medicine Laboratories, Intersocietal Commission for the Accreditation of CT Laboratories, ACR, or Joint Commission.

For SPECT/CT, conventional scanners with sodium iodide detectors or newer SPECT scanners with solid-state detectors (cadmium zinc telluride, CZT, or cesium iodide thallium, CsI [Tl]) may be used. For PET/CT devices (6), cardiac imaging may be performed in the 2-dimensional (septa in) or 3-dimensional (septa out) mode. Scanners with any of the available crystals-bismuth germanate, gadolinium oxyorthosilicate, lutetium oxyorthosilicate, or lutetium yttrium orthosilicate-can be used successfully for cardiac imaging (6).

For hybrid imaging systems, the CT configuration can be low-resolution CT (nondiagnostic CT) or 2-slice to $\geq 64$ slice multidetector-row CT. Any of these systems can be used for attenuation correction of MPI. For coronary artery calcium scoring, at least 4 -slice CT is required ( $\geq 6$ slice recommended). For coronary CTA, at least a 16-slice scanner is required ( $\geq 64$-slice multidetector-row CT recommended), with imaging capability for slice width of $0.4-0.6 \mathrm{~mm}$ and temporal resolution of $500 \mathrm{~ms}$ or less ( $\leq 350 \mathrm{~ms}$ is preferred) $(13,28,29)$.

\section{TABLE 1}

Technologist Training Considerations for Hybrid Cardiac SPECT/CT and PET/CT

Technical aspects: hands-on training for cardiac CT and MPI

Scan patient from console room and communicate with patient using intercom in scanner

Learn acquisition and processing protocols for attenuation correction, coronary artery calcium scoring, and coronary CTA

Understand concepts of prospective ECG-triggered axial or helical scans vs. retrospective ECG-gated coronary CTA

Learn operation of power injector and remote operation of dual-chamber contrast and saline injector system for coronary CTA studies

Understand interference from metallic objects and CT-based attenuation correction algorithms

Learn to minimize radiation dose from $C T$ scans

Recognize that CTAC for MPI is of low dose without need to increase dose on basis of body mass index

Learn to prepare, position, perform, and reconstruct MPI on hybrid scanners (SPECT/CT and PET/CT)

Learn to register MPI and CTAC images
Education: didactic learning

Cardiovascular anatomy and physiology

Basics of pharmacology

Radiation safety

$\mathrm{CTA}=\mathrm{CT}$ angiogram; MPI = myocardial perfusion imaging; CTAC $=$ CT for attenuation correction. 
A dual power injector for administration of iodinated contrast material and normal saline chaser is required for coronary CTA studies $(28,29)$.

Dedicated workstations for reviewing the respective MPI and CT images are required. Dedicated software for fusion of the MPI and CT images is also needed. Systems that can archive large imaging datasets, and networks capable of transferring large imaging datasets (list-mode data and cardiac CT data), are recommended.

\section{PROCEDURE/SPECIFICATIONS OF THE EXAMINATION}

\section{A. Patient screening}

Patient screening should be performed by or under the supervision of a qualified physician. The test indications must be carefully scrutinized and the test deemed necessary for patient management. The indications for the radionuclide and for the cardiac CT studies should be reviewed separately. In female patients of child-bearing potential, whether a urine or serum pregnancy test is needed is based on individual institutional policies. Contraindications for rest and stress MPI, coronary artery calcium scoring, and coronary CTA are listed in Table 2.

\section{B. Patient preparation and precautions}

Patient preparation for MPI using hybrid devices is the same as that for dedicated nuclear scanners and has been previously outlined $(2,3,5,6)$.

1. NPO: Typically, patients need to fast and take nothing

by mouth (NPO) for at least $4 \mathrm{~h}$.
2. Exercise: For MPI studies, patients must have no contraindications to the performance of exercise or pharmacologic stress testing.

3. Caffeine: For MPI and coronary CTA studies, caffeinecontaining products (coffee, tea, soda, caffeine-containing medications, diet medications, and energy drinks) must not be consumed for at least $12 \mathrm{~h}$ before the procedure.

4. Contrast allergy: For coronary CTA studies, patients with a history of mild allergy to iodinated contrast material may be pretreated. For example, one may use prednisone, $50 \mathrm{mg}$ by mouth, 13, 7, and $1 \mathrm{~h}$ before contrast injection, along with diphenhydramine, $50 \mathrm{mg}$ by mouth, $1 \mathrm{~h}$ before contrast administration $(28,30)$. For patients with a history of anaphylaxis to iodinated contrast material, alternative tests should be considered.

5. Intravenous access: For coronary CTA studies, a largegauge peripheral intravenous access line (at least 20gauge, but ideally 18-gauge) is placed, preferably in a right antecubital vein. For MPI and coronary artery calcium scoring, a small-gauge intravenous line suffices.

6. Breathing instructions: Patients should be able to cooperate with the breathing instructions for the coronary artery calcium score and coronary CTA studies. Practice breath-holds are advisable to ensure that patients are following the instructions appropriately and that respiratory motion artifacts will be avoided.

7. Medications: A list of current medications is obtained for determining any potential interactions. If possible, it is preferable to have patients withhold $\beta$-blockers at the time of MPI $(31,32)$. Phosphodiesterase use within $24 \mathrm{~h}$

TABLE 2

Relative Contraindications and Limitations to Performance of Hybrid PET/CT and SPECT/CT Studies

\begin{tabular}{|c|c|}
\hline Modality and contraindications & Consequences \\
\hline \multicolumn{2}{|l|}{ Coronary artery calcium scoring, coronary CTA or MPI } \\
\hline Pregnancy* & Unknown effects of radiation to fetus; consider alternative tests \\
\hline \multirow{2}{*}{$\begin{array}{l}\text { Clinical instability (acute myocardial infarction, } \\
\text { decompensated heart failure, severe hypotension) }\end{array}$} & Potentially unsafe for patient \\
\hline & Patient unable to cooperate with imaging (motion artifacts) \\
\hline \multirow[t]{2}{*}{ Body habitus too large for scanner } & Imaging not possible \\
\hline & Suboptimal image quality \\
\hline Inability to obtain adequate intravenous access & Imaging not possible \\
\hline \multicolumn{2}{|l|}{ Coronary artery calcium scoring, coronary CTA } \\
\hline Atrial fibrillation or frequent atrial or ventricular ectopy ${ }^{\star}$ & Motion artifacts on images \\
\hline Inability to hold breath or follow breath-hold instructions & Motion artifacts on images \\
\hline \multicolumn{2}{|l|}{ Coronary artery calcium scoring } \\
\hline $\begin{array}{l}\text { Metallic implants (pacemaker, implantable cardioverter } \\
\text { defibrillator) }\end{array}$ & Calcium score potentially less reliable \\
\hline $\begin{array}{l}\text { Known coronary artery disease (prior myocardial infarction, } \\
\text { prior revascularization) }\end{array}$ & Not indicated clinically \\
\hline \multicolumn{2}{|l|}{ Coronary CTA } \\
\hline $\begin{array}{l}\text { Known severe allergic reaction or anaphylaxis to iodinated } \\
\text { contrast material }^{*}\end{array}$ & Allergic reaction; consider alternative tests \\
\hline Renal insufficiency & Renal insufficiency worsened with iodinated contrast material \\
\hline $\begin{array}{l}\text { Contraindications for } \beta \text {-blocker use (active bronchospasm, } \\
\text { severe aortic stenosis) }\end{array}$ & Limited image quality from motion artifacts \\
\hline \multicolumn{2}{|c|}{$\begin{array}{l}\text { *Absolute contraindications. } \\
\text { CTA = CT angiogram; MPI = myocardial perfusion imaging; CTAC = CT for attenuation correction. } \\
\text { Adapted from }(1-3,5,15,28,29) \text {. }\end{array}$} \\
\hline
\end{tabular}


(sildenafil, vardenafil) to $48 \mathrm{~h}$ (tadalafil) is a contraindication for nitroglycerin administration (33). Dipyridamole-containing medications must be withheld for $48 \mathrm{~h}$ before pharmacologic stress with adenosine, regadenoson, or dipyridamole. Use of caffeine-containing medications (including antimigraine medications) can limit vasodilation from vasodilator stress agents and potentially reduce test sensitivity for ischemia. Metformin is withheld for $48 \mathrm{~h}$ after coronary CTA and restarted after renal function has been assessed. For coronary CTA studies, patients should have no contraindication to administration of $\beta$-blockers or nitroglycerin.

8. Electrocardiogram (ECG) leads: Patients should be prepared for the stress test using radiotranslucent ECG leads whenever feasible. For MPI, coronary artery calcium scoring, and coronary CTA, the ECG tracing should be evaluated with the patient's arms raised above the head.

9. Position: Patients should be positioned in a comfortable manner appropriate for the imaging device used. For patients positioned on a horizontal bed, arms should be above the head, if tolerated by the patient. For MPI, supine (standard) or prone (optional) positioning can be used depending on local preferences (5). With some of the newer heart-specific scanners, MPI may be performed in an upright position. For cardiac CT, supine positioning is standard. Appropriate table centering within the gantry is important to allow for proper function of angular $z$-axis tube current modulation.

10. Breast feeding: See section $X$ for information on MPI in the breast-feeding patient, including recommendations on the interruption of breast feeding for various radiopharmaceutical agents.

\section{Information pertinent to the procedure}

Detailed information pertinent to the procedures has been previously described $(2,3,5,6,13,28,29)$.

1. History: A focused history should be obtained about potential contraindications (Table 2), including the presence of any metallic implants or devices, claustrophobia, body mass index, chest circumference, bra cup size, ability to lie still for the duration of the scan, and ability to raise arms above head.

2. Scan time: Patients should come prepared to spend 2.5-4 $\mathrm{h}$ for a SPECT/CT MPI study, $1-2 \mathrm{~h}$ for a PET/CT MPI study, and an additional hour for the coronary CTA examination (if performed the same day).

3. Breath-hold: For a coronary artery calcium score or coronary CTA study, breath-hold techniques and shallow tidal breathing instructions should be provided. Patients should be instructed to not move during the MPI or CT procedure. Practice of breath-holding will decrease the risk of motion blurring.

4. Informed consent: Informed consent should be obtained in accordance with local institutional policies.

\section{Radiopharmaceuticals}

The most commonly used SPECT radiopharmaceuticals

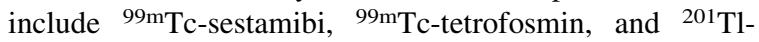
chloride $\left({ }^{201} \mathrm{Tl}\right) .{ }^{99 \mathrm{~m}} \mathrm{Tc}$-based agents have superior image quality (lower scatter) and lower radiation dose per unit administered activity. ${ }^{201} \mathrm{Tl}$ was more commonly used be- fore the development of ${ }^{99 \mathrm{~m}} \mathrm{Tc}$ agents but continues to be used, particularly when ${ }^{99 \mathrm{~m}} \mathrm{Tc}$ agents are in short supply. Details of radiopharmaceuticals, dosages, and specific image acquisition parameters for SPECT have been previously outlined $(2,3,5)$. The most common clinically used PET radiopharmaceuticals include ${ }^{82} \mathrm{Rb},{ }^{13} \mathrm{~N}$-ammonia for MPI, and ${ }^{18} \mathrm{~F}$-fluorodeoxyglucose $\left({ }^{18} \mathrm{~F}\right.$-FDG) for metabolic imaging. ${ }^{82} \mathrm{Rb}$ is generator-produced and widely available for clinical use. ${ }^{13} \mathrm{~N}$-ammonia is a cyclotron-produced radiotracer available in hospitals with an onsite cyclotron. Details of radiopharmaceuticals, dosages, and specific image acquisition parameters for PET have been previously outlined (6).

\section{E. Acquisition protocols}

This section describes the acquisition protocols for SPECT/CT MPI, PET/CT MPI, ${ }^{18}$ F-FDG PET/CT, cardiac $\mathrm{CT}$, and fusion of radionuclide and $\mathrm{CT}$ images.

\section{SPECT/CT MPI}

Perfusion imaging can be performed with ${ }^{99 \mathrm{~m} T c}$ agents or ${ }^{201} \mathrm{Tl}$ using previously described protocols (5). SPECT is standard, with planar imaging limited to the imaging of claustrophobic patients and patients with a large body habitus (patients above the weight limit of the imaging table or not able to fit within the scanner gantry). Gated SPECT is recommended whenever feasible.

For ${ }^{99 \mathrm{~m}} \mathrm{Tc}$, the most commonly used protocols are a 1-d, low-dose rest and high-dose stress protocol (Table 3) or a 2-d protocol (for patients with a high body mass index) with equal radiotracer injection $(925-1,110 \mathrm{MBq}$ [25-30 mCi] of ${ }^{99 \mathrm{~m}} \mathrm{Tc}$ ) (5). A protocol using ${ }^{201} \mathrm{Tl}$ at rest and ${ }^{99 \mathrm{~m}} \mathrm{Tc}$ perfusion tracers during stress, the so-called dual-isotope technique, allows for a shorter study but at the expense of higher radiation dose. ${ }^{201} \mathrm{Tl}$ MPI with 4and 24-h redistribution imaging is also used for assessment of myocardial viability. If ${ }^{99 \mathrm{~m}} \mathrm{Tc}$ isotope is not available, ${ }^{201} \mathrm{Tl}$ is an alternate agent for rest and stress MPI.

Stress-only imaging with ${ }^{99 \mathrm{~m}} \mathrm{Tc}$ may reduce test duration, radiation dose, and costs. Preliminary evidence suggests normal stress-only SPECT to be equivalent to normal rest and stress MPI for risk stratification in low-risk patients $(34,35)$. Stress-only MPI can be considered as an option by experienced and well-qualified physicians in highly selected patients with low risk or very low risk in whom the stress images are anticipated to be normal (36).

A typical SPECT protocol is shown in Table 3 (5). Patients are scanned supine (standard), prone (optional), or upright depending on local preferences and scanner type. SPECT/CT frequently uses an acquisition protocol that has a $128 \times 128$ matrix with a zoom factor of 1.0 . The CT protocols for SPECT/CT and PET/CT are described in part E4 of this section (5).

\section{PET/CT MPI}

MPI $\left({ }^{13} \mathrm{~N}\right.$-ammonia or $\left.{ }^{82} \mathrm{Rb}\right)$ and myocardial metabolic imaging ( $\left.{ }^{18} \mathrm{~F}-\mathrm{FDG}\right)$ can be performed using PET (6). Details of ${ }^{13} \mathrm{~N}$-ammonia imaging protocols have been previously published ( 6 ).

${ }^{82} \mathrm{Rb}$ is the most commonly used radiotracer for PET MPI. Patients are typically scanned supine with the arms raised above the shoulders. The heart position is localized using a scout CT image (10 mA, $120 \mathrm{keV})$ followed by CTAC (10-20 mA, $120 \mathrm{keV})$ for attenuation correction 
TABLE 3

Sample Protocol for Single-Day Rest-Stress ${ }^{99 m}$ Tc-Labeled Scans with SPECT MPI

\begin{tabular}{|c|c|c|c|}
\hline Feature & Rest study & Stress study & Technique \\
\hline Dose & 296-444 MBq (8-12 mCi*) & 890-1,330 MBq (24-36 mCi*) & Standard \\
\hline \multirow[t]{3}{*}{ Position } & Supine & Supine & Standard \\
\hline & Prone & Prone & Optional \\
\hline & Upright/semiupright & Upright/semiupright & Optional \\
\hline \multicolumn{4}{|l|}{ Delay time (intervals) } \\
\hline Injection to imaging & $30-60 \mathrm{~min}$ & $15-60 \mathrm{~min}$ & Standard \\
\hline Rest to stress & & $30 \mathrm{~min}$ to $4 \mathrm{~h}$ & Standard \\
\hline Energy window & 15\%-20\% symmetric & Same & Standard \\
\hline Collimator & Low energy high resolution & Same & Preferred \\
\hline Orbit & $180^{\circ}\left(45^{\circ} \mathrm{RAO}\right.$ to $\left.45^{\circ} \mathrm{LPO}\right)$ & Same & Preferred \\
\hline \multirow[t]{2}{*}{ Orbit type } & Circular & Same & Standard \\
\hline & Noncircular & Same & Standard \\
\hline \multicolumn{4}{|l|}{ Pixel size } \\
\hline 64 by 64 matrix & $6.4 \pm 0.4 \mathrm{~mm}$ & Same & Standard \\
\hline 128 by 128 matrix & $8-9 \mathrm{~mm}$ & Same & Optional \\
\hline \multirow[t]{2}{*}{ Acquisition type } & Step and shoot & Same & Standard \\
\hline & Continuous & Same & Optional \\
\hline Number of projections & $60-64^{\dagger}$ & Same & Standard \\
\hline Matrix & $60 \times 64$ & Same & Standard \\
\hline Time/projection & $25 \mathrm{~s}$ & $20 \mathrm{~s}$ & Standard \\
\hline ECG gated & Optional & Standard & Preferred \\
\hline \multirow[t]{2}{*}{ Frames/cycle } & 8 & 8 & Standard \\
\hline & 16 & 16 & Optional \\
\hline R-to-R window (for ejection fraction) & $100 \%$ & $100 \%$ & Preferred \\
\hline Attenuation correction & $\begin{array}{c}\text { Measured attenuation correction } \\
\text { before or after SPECT scan }\end{array}$ & $\begin{array}{c}\text { Measured attenuation correction } \\
\text { before or after SPECT scan }\end{array}$ & Optional \\
\hline
\end{tabular}

*Lower stress and rest doses may be used with new scanners with solid-state detectors and new software reconstruction.

${ }^{\dagger} 64$ projections are preferable for ${ }^{201} \mathrm{TI}$.

$\mathrm{RAO}=$ right anterior oblique; LPO = left posterior oblique.

Adapted and reproduced with permission from (5).

and emission imaging. For ${ }^{82} \mathrm{Rb}$ imaging, 1,480-2,040 $\mathrm{MBq}$ (40-60 mCi) (2-dimensional), 370-740 MBq (10$20 \mathrm{mCi}$ ) (3-dimensional, bismuth germanate systems), or 1,110-1,480 MBq (30-40 mCi) (3-dimensional, lutetium oxyorthosilicate systems) are injected, followed by image acquisition for $7 \mathrm{~min}$ without a prescan delay using list mode (preferred), or in gated or static mode using a prescan delay (70-90 s for left ventricular ejection fraction $>50 \%$, and 91-130 s for left ventricular ejection fraction $<50 \%)$.

Stress testing (predominantly pharmacologic stress) is performed using standard protocols and radiotracer injected at maximal hyperemia followed by the same imaging steps as described for rest MPI. Exercise stress is not frequently used but is possible with ${ }^{13} \mathrm{~N}$-ammonia and challenging with ${ }^{82} \mathrm{Rb}$.

Typically, the patient position is not changed on the scanner table between the rest and stress images, and a single CTAC study is adequate for attenuation correction of the rest and stress images. If the patient is moved after rest MPI, CTAC after stress MPI may be preferable to CTAC during peak hyperemia, particularly when vasodilator stress is used, because of respiratory stimulation caused by vasodilator agents.

List-mode emission data are unlisted into gated, static, and dynamic image sets for analysis. The images are reconstructed using filtered backprojection or iterative expectation maximization (e.g., ordered-subsets expectation maximization). The protocols for cardiac PET have been described in great detail previously (Table 4) (6).

3. ${ }^{18} \mathrm{~F}-\mathrm{FDG}$ PET/CT

Cardiac ${ }^{18} \mathrm{~F}$-FDG imaging is performed for assessment of myocardial viability and for identifying cardiac inflammation (e.g., sarcoidosis).

For myocardial viability assessment, the following steps can be followed: Perform rest MPI using ${ }^{82} \mathrm{Rb}$ or ${ }^{13} \mathrm{~N}$-ammonia as described above for PET/CT MPI. Some facilities use the SPECT/CT attenuation-corrected MPI or a gated SPECT MPI study for comparison with the ${ }^{18}$ F-FDG study. Follow this with glucose loading and insulin administration for viability assessment using standard protocols (6). Check baseline blood sugar, and perform oral glucose or intravenous glucose loading protocols as shown in Tables 5 and 6 (6). Perform ${ }^{18} \mathrm{~F}-$ FDG imaging as a gated study (whenever possible) for 1530 min using previously described parameters (Table 7). In case of high blood-pool activity on the ${ }^{18}$ F-FDG images (typically seen with insulin resistance), more intravenous insulin may be administered and images reacquired (6).

${ }^{18} \mathrm{~F}-\mathrm{FDG}$ imaging is performed for assessment of myocardial inflammation in patients with suspected or known cardiac sarcoidosis (37-40). Preliminary retrospective 
TABLE 4

Imaging Protocol for ${ }^{82} \mathrm{Rb}$ PET MPI

\begin{tabular}{|c|c|c|}
\hline \multicolumn{2}{|l|}{ Feature } & \multirow{2}{*}{$\frac{\text { Technique }}{\text { Standard }}$} \\
\hline Stress testing & Pharmacologic agents & \\
\hline Tracer dose & & Standard \\
\hline 2-dimensional scanner & 1,480-2,220 MBq (40-60 mCi) & Standard \\
\hline \multirow[t]{2}{*}{ 3-dimensional scanner } & $370-740 \mathrm{MBq}(10-20 \mathrm{mCi})$ & Standard \\
\hline & $2,220-1,480 \mathrm{MBq}(30-40 \mathrm{mCi})$ for lutetium oxyorthosilicate systems & \\
\hline Injection rate & Bolus of $\leq 30 \mathrm{~s}$ & Standard \\
\hline \multirow[t]{3}{*}{ Imaging delay after injection } & LVEF > 50\%: 70-90 s & Acceptable \\
\hline & LVEF $\leq 50 \%$ or unknown: $90-130 \mathrm{~s}$ & \\
\hline & List mode: acquire immediately & \\
\hline Patient positioning & & Standard \\
\hline \multirow{2}{*}{ PET } & Scout scan: $370-740 \mathrm{MBq}(10-20 \mathrm{mCi}){ }^{82} \mathrm{Rb}$ & \\
\hline & Transmission scan & Optional \\
\hline $\mathrm{PET} / \mathrm{CT}$ & CT scout & Standard \\
\hline \multirow[t]{2}{*}{ Imaging mode } & List mode/gated/dynamic (no delay after injection) & Preferred \\
\hline & Gated (delay after injection) & Optional \\
\hline \multirow[t]{2}{*}{ Imaging duration } & $3-6 \min$ & Standard \\
\hline & $3-10 \mathrm{~min}$ & Optional \\
\hline Attenuation correction & Measured attenuation correction, before or after & Standard \\
\hline Reconstruction method & FBP or iterative expectation maximization (OSEM) & Standard \\
\hline Reconstruction filter & Sufficient to achieve desired resolution/smoothing, matched stress to rest & Standard \\
\hline Reconstructed pixel size & $2-3 \mathrm{~mm}$ & Preferred \\
\hline
\end{tabular}

studies suggest high accuracy and superiority compared with ${ }^{67} \mathrm{Ga}$ imaging (38). Fasting ${ }^{18} \mathrm{~F}$-FDG imaging may result in variable physiologic myocardial glucose uptake. Hence, to ensure uptake by inflamed tissue but optimize suppression of ${ }^{18} \mathrm{~F}$-FDG uptake by normal myocytes (reduce nonspecific uptake), patients are instructed to take a diet rich in fats with no carbohydrates for 12-24 h before the test or fast for 12-18 h and/or use intravenous heparin, $15-50$ units $/ \mathrm{kg}$, about $15 \mathrm{~min}$ before the injection of ${ }^{18} \mathrm{~F}-$ FDG $(40,41)$. A rest MPI study (SPECT or PET, prefera- bly a gated scan) is first performed. This is followed by the intravenous administration of ${ }^{18} \mathrm{~F}-\mathrm{FDG}$ (370-555 MBq [10$15 \mathrm{mCi}]$ ), a 90-min uptake period, and subsequent image acquisition in a static mode using parameters described in Table 7. Whole-body ${ }^{18} \mathrm{~F}-\mathrm{FDG}$ imaging is performed from the cerebellum to the upper thigh to be able to assess ${ }^{18} \mathrm{~F}$ FDG uptake in extracardiac regions (neck, mediastinum, lungs, and abdominal lymph nodes). The rest myocardial perfusion and cardiac ${ }^{18} \mathrm{~F}-\mathrm{FDG}$ images are reconstructed and reoriented into standard cardiac planes (short axis,

TABLE 5

Cardiac ${ }^{18}$ F-FDG Preparation Guidelines

\begin{tabular}{|c|c|c|}
\hline Step & Procedure & Technique \\
\hline \multirow[t]{2}{*}{ Fasting period } & Instruct patient to fast $6-12 \mathrm{~h}$ (preferred) or $<6 \mathrm{~h}$ (suboptimal) & \\
\hline & Check blood glucose & \\
\hline \multirow[t]{8}{*}{ Oral glucose loading } & IF: fasting blood glucose < $110 \mathrm{mg} / \mathrm{dL}$ (6.11 mmol/L) & Standard \\
\hline & AND: no known diabetes & \\
\hline & THEN: & \\
\hline & Administer oral glucose load (typically 25-100 g; Table 6) & \\
\hline & Monitor blood glucose (Table 6) & \\
\hline & IF: fasting blood glucose $>110-130 \mathrm{mg} / \mathrm{dL}$ (6.11-7.22 mmol/L) & Standard \\
\hline & OR: known diabetes & \\
\hline & THEN: see Table 6 & \\
\hline \multicolumn{3}{|l|}{ OR: } \\
\hline Intravenous dextrose infusion & See reference $(6)$ & Optional \\
\hline Acipimox & 250 mg orally (not available in United States) & \\
\hline${ }^{18} \mathrm{~F}-\mathrm{FDG}$ intravenous injection & Timing is dependent on which option was selected (Table 7 , item 1) & Standard \\
\hline PET & 45-90 min after ${ }^{18} \mathrm{~F}-\mathrm{FDG}$ injection, start imaging (Table 7) & \\
\hline \multicolumn{3}{|c|}{ Reproduced with permission from (6). } \\
\hline
\end{tabular}


TABLE 6

Guidelines for Blood Glucose Maintenance (e.g., After Oral Glucose Administration)

\begin{tabular}{cc}
\hline $\begin{array}{c}\text { Blood glucose at } 45-90 \\
\text { min after administration }\end{array}$ & Possible restorative measure \\
\hline $130-140 \mathrm{mg} / \mathrm{dL}$ & $1 \mathrm{U}$ regular insulin intravenously* \\
$(7.22-7.78 \mathrm{mmol} / \mathrm{L})$ & \\
$140-160 \mathrm{mg} / \mathrm{dL}$ & $2 \mathrm{U}$ regular insulin intravenously \\
$(7.78-8.89 \mathrm{mmol} / \mathrm{L})$ & \\
$160-180 \mathrm{mg} / \mathrm{dL}$ & $3 \mathrm{U}$ regular insulin intravenously \\
$(8.89-10 \mathrm{mmol} / \mathrm{L})$ & \\
$180-200 \mathrm{mg} / \mathrm{dL}$ & $5 \mathrm{U}$ regular insulin intravenously \\
$(10-11.11 \mathrm{mmol} / \mathrm{L})$ & \\
$>200 \mathrm{mg} / \mathrm{dL}$ & Notify physician \\
$(>11.11 \mathrm{mmol} / \mathrm{L})$ & \\
\hline
\end{tabular}

*Optional; may be considered if total amount of insulin administered is low.

Reproduced with permission from (6).

vertical long axis, and horizontal long axis) for interpretation. The whole-body ${ }^{18}$ F-FDG images should be interpreted by trained nuclear medicine physicians or physicians credentialed to supervise and interpret body PET/CT $(42,43)$.

4. Attenuation-correction CT scan (CTAC)

Three types of CT protocols are used for hybrid imaging procedures: an unenhanced, nongated, free tidal breathing CT scan (5-mm slice thickness) for attenuation correction; an unenhanced, gated, breath-hold CT scan (2- to 3-mm slice thickness) for coronary artery calcium scoring; and a contrast-enhanced, gated, breath-hold CT scan (0.5- to $0.75-\mathrm{mm}$ slice thickness) for coronary CTA.

Several years ago, CT was incorporated into hybrid devices solely for the purposes of attenuation correction (44). The main advantage of these initial devices was that the CT image was of low temporal and spatial resolution and more comparable with the resolution of the radionuclide scans, and may result in fewer misregistration artifacts. However, the scan duration was prolonged and the limited CT was not helpful for anatomic disease localization, particularly for general nuclear medicine applications. Subsequently, diagnostic CT scanners (2-, 4-, 6-, 8-, and 64-slice multidetector-row CT) were integrated with SPECT or PET scanners, allowing the acquisition of standard multidetector CT scans for attenuation correction, coronary artery calcium scoring, and coronary CTA, at a fast pace. These scanners produce high-resolution CT scans in approximately $10 \mathrm{~s}$. Because of the faster imaging in relation to the emission scans, the heart position is averaged over a few breathing cycles, compared with SPECT or PET, for which the heart position is averaged over $5-12 \min (6)$. This has resulted in a relatively higher frequency of misregistration artifacts and the need to consider various protocols to coregister the PET (or SPECT) and the CT images.

Investigators experimented with various breathing techniques such as free tidal breathing, breath-hold at end inspiration, or breath-hold at end expiration (45). Currently, free tidal breathing with averaging of respiratory movement appears to be the best technique (6). Lowdose or ultra-low-dose CT $(46,47)$, as well as slow CT $(48,49)$ and ultra-fast CT, have been tried to match the image resolution to that of the MPI in an attempt to improve registration of the MPI and CT images, but with no distinct advantages. Dual gated PET/CT with ECG gating and gating for respiratory motion are feasible and are being tested (50-52). Some of the newer-generation fast SPECT scanners have low-dose CT-based attenuation correction (53). General guidelines for CT-based transmission imaging are listed in Table 8.

Transmission scanning with shallow tidal breathing is the best protocol. Tube current and voltage of approximately $10-20 \mathrm{~mA}$ and $80-140 \mathrm{kVp}$ are recommended. For PET MPI, a single CTAC is standard (since the patient can undergo stress agent infusion in the PET gantry). However, because of frequent patient motion between the separately acquired MPI and CTAC studies, which can be difficult to detect by patient observation, or if the patient is moved between the rest and stress MPI, a separate CTAC for the rest and stress MPI study may be used (optional). Some scanners will not allow the use of CTAC for attenuation correction if the patient or table position has shifted by more than $5 \mathrm{~mm}$ between trans-

TABLE 7

${ }^{18}$ F-FDG Cardiac PET Acquisition Parameters

\begin{tabular}{ll}
\hline \multicolumn{1}{c}{ Parameter } & \multicolumn{1}{c}{ Recommendation } \\
\hline Tracer dose & $185-370 \mathrm{MBq}(5-10 \mathrm{mCi}), 3-$ dimensional mode \\
& $370-555 \mathrm{MBq}(10-15 \mathrm{mCi}), 2-$ dimensional mode \\
\hline Delay after injection & $45-60 \mathrm{~min}$ (nondiabetic patients) \\
& $60-90 \mathrm{~min}$ (diabetic patients) \\
\hline Patient positioning & Supine (arms-up preferred) \\
Imaging mode & 2 -dimensional or 3-dimensional; list, gated, or static \\
\hline Image duration & $10-30$ min (depending on counting rate and dose) \\
\hline Attenuation correction & Before or immediately after emission scan using radionuclide or CT transmission imaging \\
Reconstruction method & Filtered backprojection or ordered-subsets expectation maximization (iterative reconstruction) \\
\hline Reconstructed pixel size & $2-5$ mm \\
\hline Adapted with permission from (6). & \\
\hline
\end{tabular}


TABLE 8

Guidelines for CT-Based Transmission Imaging

\begin{tabular}{|c|c|c|}
\hline CT parameter & General principle & Effect on patient dose \\
\hline Slice collimation & $\begin{array}{l}\text { Collimation should approximate slice thickness } \\
\text { of PET (e.g., } 4-5 \mathrm{~mm} \text { ) }\end{array}$ & No effect \\
\hline Gantry rotation speed & $\begin{array}{l}\text { Slower rotation helps blur cardiac motion (e.g., } \\
1 \mathrm{~s} / \text { revolution or slower) }\end{array}$ & $\begin{array}{l}\text { Increased radiation with slower } \\
\text { gantry rotation }\end{array}$ \\
\hline Table feed per gantry rotation (pitch) & Pitch should be relatively high (e.g., 1:1) & Inversely related to pitch \\
\hline ECG gating & ECG gating is not recommended & Decreases without ECG gating \\
\hline Tube potential & $\begin{array}{l}80-140 \mathrm{kVp} \text { is used, depending on manufacturer } \\
\text { specification }\end{array}$ & Increases with higher kVp \\
\hline Tube current & $\begin{array}{l}\text { Because scan is acquired only for attenuation } \\
\text { correction, low tube current is preferred } \\
(10-20 \mathrm{~mA})\end{array}$ & Increases with higher $\mathrm{mA}$ \\
\hline Breathing instructions & $\begin{array}{l}\text { End-expiration breath-hold or shallow free } \\
\text { breathing is preferred }\end{array}$ & No effect \\
\hline Reconstructed slice thickness & $\begin{array}{l}\text { Thickness should approximate slice thickness } \\
\text { of SPECT or PET (e.g., } 4-7 \mathrm{~mm} \text { ) }\end{array}$ & No effect \\
\hline
\end{tabular}

mission and emission imaging. For SPECT MPI, a separate $\mathrm{CT}$ scan is typically necessary for the rest and stress MPI studies.

The use of the transmission CT scan to assess for coronary artery calcium can approximate the extent of coronary calcification (54). This approach, however, does not provide an accurate measure of coronary artery calcium, since it is a nongated study that has artifacts due to coronary artery motion. Also, the attenuation scan is obtained with a much lower photon density than the calcium score scans. Frequently, the use of CTAC for visual assessment of coronary artery calcium may fail to detect small amounts of calcium in approximately $8 \%$ of patients (54).

The use of the coronary artery calcium score CT scan for attenuation correction $(55,56)$ has been explored. However, the calcium score is an end-diastolic image obtained typically during an inspiratory breath-hold and may not register adequately with the MPI, which is an averaged image of the heart over several cardiac cycles obtained during shallow tidal breathing.

5. Coronary artery calcium scan

The coronary artery calcium score CT scan is performed per standard protocols as described previously (28). Scanners with excellent spatial and temporal resolution must be used to ensure good image quality (see section VIII, Equipment Specification) $(13,28)$.

An unenhanced, prospectively ECG-triggered axial acquisition is reconstructed at a slice thickness of 2.5 or $3 \mathrm{~mm}$ at $65 \%-80 \%$ of the R-R cycle during an inspiratory breath-hold. To reduce radiation dose, it may be beneficial to use prospective triggering (depending on the scanner type) $(56,57)$, wherein the $\mathrm{x}$-ray tube is activated only during a prespecified phase of the cardiac cycle (as opposed to retrospective gating), thereby reducing dose delivered to the patient.

Although, theoretically, the imaging parameters might be adjusted on the basis of body habitus to lower radiation dose (lower tube current or voltage in patients with a smaller body mass index), only the standard tube potential $(\mathrm{kVp})$ and current $(\mathrm{mA})$ settings are currently recommended for accurate coronary artery calcium scoring. Preliminary data suggest that a tube potential of $100 \mathrm{kVp}$ can reliably measure the coronary artery calcium score; however, the approach requires a different calcium threshold from the $130 \mathrm{HU}$ used with standard calcium scoring algorithms and may vary with scanners. Although of potential use in reducing radiation dose, the use of lower tube potential has not yet been validated, and no standard threshold has been established for coronary artery calcium scoring (58).

Retrospective gating with helical imaging is not suggested, because of increased radiation burden to the patient. A prospectively triggered mode without overlapping slices (step-and-shoot acquisition) has been shown to reduce radiation dose significantly, when compared with a helical mode with overlapping images $(29,59)$. High heart rates may degrade image quality. However, $\beta$-blockers are not routinely used before coronary artery calcium scans.

6. Coronary CTA

Listed below are suggested guidelines for the performance of coronary CTA; however, these parameters and guidelines may vary with patient cardiac output, heart rate, and rhythm, as well as the type of scanner, vendor, and platform). Certain technical considerations need to be considered for hybrid coronary CTA and MPI studies. The scanners used must have an excellent spatial and temporal resolution to ensure image quality (see section VIII, Equipment Specifications) $(13,28,29)$.

Patient preparation. A large-bore intravenous needle is required for the coronary CTA study (to rapidly inject iodinated contrast material at $4-7 \mathrm{~mL} / \mathrm{min}$ ), ideally in the anticubital fossa. The wrist is to be avoided because of the risk of extravasation. If the study is scheduled as a combined study with rest and stress MPI, it is preferable to perform the MPI scan before the CT scan (to minimize 
any potential interference in attenuation correction from the iodinated contrast material). For pharmacologic studies, vasodilator stress is preferred to dobutamine stress because of the increased heart rate from dobutamine infusion.

Oral or intravenous $\beta$-blockers are routinely used before coronary CTA to bring the heart rate to the target of 50-60 bpm. Because the temporal resolution of the scanners is limited, motion artifacts are minimized at heart rates of less than $60 \mathrm{bpm}$. $\beta$-blocker administration may less frequently be necessary when dual-source CT technology of a higher temporal resolution becomes incorporated into hybrid scanners. Nitroglycerin $(0.4 \mathrm{mg}$ sublingually or 1 spray) is frequently used immediately before the coronary CTA study to improve visualization of smaller-caliber coronary vessels through vasodilation).

Iodinated contrast material with maximal iodine concentration vasodilation ( $\geq 300 \mathrm{mg} \mathrm{I} / \mathrm{mL}$ ) is preferred. Whether isoosmolar contrast agents are superior to higher-osmolar contrast material for the prevention of nephrotoxicity is still a matter of debate. Contrast material is administered intravenously at a rate of $4-7 \mathrm{~mL} / \mathrm{s}$. The amount of contrast material used varies $(50-120 \mathrm{~mL})$ on the basis of the selected flow rate, the scan length in the $z$-dimension, the type of scanner, and the selected scan mode (retrospectively ECG-gated, prospectively triggered axial, or prospectively triggered high-pitch spiral-scan mode).

Technical details. The coronary CTA scan on hybrid devices is acquired using standard protocols $(13,28,29)$. To reduce radiation dose, it is important to adjust the dose on the basis of body habitus. Reducing tube potential from $120 \mathrm{kV}$ to $100 \mathrm{kV}$ should be considered in individuals who weigh less than $85 \mathrm{~kg}$ or have a body mass index less than $30 \mathrm{~kg} / \mathrm{m}^{2}$, and reduction to $80 \mathrm{kV}$ can be considered in thinner patients (60). A higher tube current and voltage may be considered in obese individuals to improve signal-to-noise ratio. If retrospective gating is used, ECG dose modulation with a low tube current during systolic phases is recommended $(28,29,60)$.

Prospective triggering techniques reduce radiation dose $(59,61,62)$. Instead of a low-pitch helical mode with overlapping images, a prospectively ECG-triggered scan using an axial (step-and-shoot) acquisition may reduce dose significantly while maintaining image quality $(62,63)$. This approach is applicable only when the patient's heart rate is sufficiently slow $(<60 \mathrm{bpm})$ and there is no arrhythmia. Widening of the acquisition window (padding) (the interval when the $\mathrm{x}$-ray tube is activated) must be minimized to keep radiation dose low (64). Prospective triggering obviates the possibility of evaluating LV function with gated CT.

Novel techniques to further minimize the radiation dose from coronary CTA using prospective ECG-triggered high-pitch spiral acquisition (65) or adaptive statistical iterative reconstruction algorithms $(66,67)$ are evolving. To optimize the scan duration with hybrid ${ }^{82} \mathrm{Rb} \mathrm{PET} /$ CTA, a stress-first protocol with vasodilator stress may be considered. $\beta$-blockers can be administered intravenously (metoprolol, $5 \mathrm{mg}$, repeated at 5-min intervals) after the stress ${ }^{82} \mathrm{Rb}$ MPI is completed.
Coronary CTA steps $(13,28,29)$. Each step in coronary CTA is performed using breath-hold instructions to train the patient. A scout scan of the chest is performed to localize the heart position. This scan may be used to define the start and end locations for the coronary CTA study.

The coronary CTA scan length ranges from 2.5 to 5.0 $\mathrm{cm}$ (1-2 in) above the aortic root/left main takeoff and from 2.5 to $5.0 \mathrm{~cm}$ (1-2 in) below the bottom of the heart silhouette as determined by the scout image. Since the scan length determines the duration of breath-hold and radiation dose to patients, the minimal required scan length should be used. However, if the scan length is too tight, there is a risk of not including the take-off of the left main coronary artery or the distal posterior descending artery. Frequently, the carina is used as a cranial landmark because it is readily identifiable on the topographic scout image and avoids the risk of exclusion of the left main or left anterior descending coronary arteries.

The arrival of contrast material in the ascending aorta is timed using either a timing bolus scan or a bolus tracking acquisition for automatic scan triggering. For a timing bolus scan, $10-20 \mathrm{~mL}$ of iodinated contrast material are injected at 4-6 mL/s using the power injector, followed by approximately $20 \mathrm{~mL}$ of normal saline chaser. Scanning is started (10 s later) as a single CT slice at $2 \mathrm{~cm}$ above the aortic root, 1 image every $2 \mathrm{~s}$. The aorta is observed for contrast opacification, and the acquisition is stopped when a decrease in opacification is observed. The images are reviewed visually (optional) or using a program (standard) to identify the slice with maximal contrast and to calculate the time to peak opacification (including the 10-s delay). Once the time to peak contrast opacification in the aorta is determined, an additional $3-4 \mathrm{~s}$ is added to allow for opacification of the coronary arteries. This procedure offers the advantages of testing the intravenous line, avoids the risk of a delayed or early start of the imaging, and provides a chance to observe patient cooperation with instructions and breath-holding (28).

A bolus tracking acquisition for automatic scan triggering is based on a prespecified intensity of contrast enhancement in the ascending or descending aorta (e.g., $100 \mathrm{HU})$. In this mode, the gantry is stationary and performs repeated axial scans through the aorta. The CTA acquisition is triggered when a region of interest over the aorta reaches the predetermined contrast enhancement threshhold.

A dual-head power injector is loaded with contrast in one syringe and normal saline in the other. Typical contrast injection rates range from 4 to $7 \mathrm{~mL} / \mathrm{s}$, dependent on body habitus and cardiac output. Higher rates of 7-8 mL may occasionally be used in individuals with a high cardiac output.

Typically, $50-120 \mathrm{~mL}$ of contrast material is administered, followed by an approximately $40-\mathrm{mL}$ normal-saline flush during an inspiratory breath-hold. The precise amount of contrast material is based on scan duration (which is based on the prescribed start and end locations of the scan) and other parameters as described previously. 
The saline chaser is used to keep a tight bolus of contrast material and to clear the material out of the right heart to avoid streak artifacts on the right coronary artery.

Images are reconstructed using standard parameters to the thinnest possible slice $(0.5-0.75 \mathrm{~mm}$, determined by the scanner detector configuration), with an increment resulting in an overlap of approximately one-third slice (enhances resolution in the $z$-dimension). In patients with a high body mass index, a slightly thicker slice $(1 \mathrm{~mm})$ may be used to avoid images with poor signal-to-noise ratio. Scanners with editing capabilities may use ECG editing to improve cardiac motion from an occasional ectopic beat during the acquisition. Images may be reconstructed in a larger field of view to study and interpret extracardiac findings.

7. Fusion of radionuclide and CT images

The radionuclide and CT images are acquired sequentially and can be displayed separately or as fused images (overlay of radionuclide and CT images) using hardware or software coregistration techniques. Images are not acquired in a fused mode. Management decisions are typically made using the integrated diagnostic information from radionuclide (SPECT or PET) and CT without actual image fusion, along with the fused images.

\section{F. Integrating diagnostic information from radionuclide and $\mathrm{CT}$ images}

Integration of the radionuclide and CT information and image fusion are new techniques whose clinical role is evolving. Literature to support their clinical utility is growing, but definitive studies are limited. Literature is evolving to support the diagnostic and prognostic role of calcium scoring in patients without known coronary artery disease (68-83). Likewise, several studies support the complementary roles of the diagnostic and prognostic information provided by CTA and MPI in symptomatic individuals (84-97). A joint position statement on hybrid cardiac imaging was published by the European Association of Nuclear Medicine, the European Society of Cardiac Radiology, and the European Council of Nuclear Cardiology (11).

1. Coronary artery calcium scoring and MPI

Several studies (68-73) have shown that coronary artery calcium scoring has incremental diagnostic value over MPI because of its ability to quantify overall atherosclerotic burden. Depending on the population studied, about $21 \%-47 \%$ of patients with normal MPI results have a high coronary artery calcium score (Agatston score $>400)(68,70,74)$. Preliminary data suggest that when available, the combined information of MPI and coronary artery calcium score may be used to manage the aggressiveness of coronary risk factor modification $(69,73,82)$. Studies have reported that from $15 \%$ to $40 \%$ of patients with an Agatston score of more than 400 demonstrate stress-induced ischemia on MPI $(68,70,71)$. A normal MPI study is associated with a low event rate for 2-3 y even in the presence of extensive coronary artery calcification (79). However, a high calcium score may identify a higher risk in symptomatic subjects and during longterm follow-up $(81,98,99)$. These findings suggest the safety of an aggressive medical management strategy with repeated MPI in 2-3 y, as well as caution regarding referral to invasive coronary angiography.

Acquisition protocols for coronary artery calcium scoring and MPI are described in part $\mathrm{E}$ of this section. Coronary artery calcium scoring and MPI images are interpreted separately and do not require a fusion display of the images. Of note, coronary artery calcium scoring does not assess the severity of coronary artery stenosis (100). Also, calcium scoring may be unreliable when used alone for risk assessment of patients with acute symptoms, especially women and younger patients (101), probably because noncalcified plaque (often the culprit lesion in acute coronary syndromes) is not identified.

2. Coronary CTA and MPI

The combined diagnostic information from CTA with MPI can be complementary in specific clinical scenarios (84-97,102). Multivessel and subclinical atherosclerosis can be diagnosed accurately with CTA and add to the diagnostic value of MPI. The negative predictive value of CTA to exclude obstructive epicardial CAD is excellent (103). However, the positive predictive value of CTA for identifying hemodynamically significant ischemia territories is only modest (103). Also, stenosis severity can be overestimated by CTA (104), especially in patients with beam-hardening artifacts from calcified coronaries. The magnitude of ischemic burden on rest and stress MPI determines the hemodynamic significance of coronary artery stenoses (1) and predicts benefits from revascularization $(105,106)$. Also, the distal coronary segments or coronary segments obscured by multidetector-row CT artifacts can be well studied with MPI. In one study with hybrid PET MPI and concomitant CTA (85), only $47 \%$ of significant angiographic stenoses were associated with ischemia and about half the patients with normal MPI results had evidence of non-flow-limiting CAD. Discordant findings on MPI and CTA can result from microvascular dysfunction (abnormal blood flow without obstructive epicardial CAD), calcified and nonobstructive $\mathrm{CAD}$ with normal perfusion or obstructive CAD that is not flow-limiting (due to hemodynamic or collateral changes), and imaging artifacts $(7,107-110)$. These findings should be interpreted and reported in the context of the combined imaging data (see section VII). Reduced left ventricular ejection fraction, an important predictor of outcomes after revascularization (111), can be determined by MPI (112-114). Therefore, combined MPI and CTA can provide better characterization of the extent and severity of underlying $\mathrm{CAD}$ and potential benefit from revascularization than does either technique alone. However, although there are several studies showing the complementary value of combined MPI and CTA, this is an evolving field with ongoing studies (115) and definitive data are awaited.

3. Indications and appropriate use criteria for hybrid imaging Addressing the indications and appropriate use criteria for imaging with hybrid devices is not the primary focus of this document. The indications and appropriate use criteria for MPI using hybrid devices are the same as for dedicated SPECT or PET scanners and have been previously addressed $(1,4)$. Hybrid imaging is a relatively novel field, 
and there are no well-accepted indications for combined MPI and coronary artery calcium scoring or coronary CTA. In asymptomatic patients with an abnormal coronary artery calcium score, MPI may be appropriate for further evaluation if the Agatston score is more than 400 or, in patients with a high CHD risk, is more than 100 but not higher than 400 (4). Patients with an uninterpretable or equivocal MPI study may be candidates for CTA and vice versa (4). The indications must be closely scrutinized on a case-by-case basis.

Because of the additional radiation burden and multiple testing, the results of the first test (CTA or MPI) must be reviewed to assess the need for the second test. If the first test is normal, the second test is typically not indicated. Therefore, combined MPI and coronary CTA would be the approach best suited presently for research laboratories or for laboratories with a low volume of studies, permitting careful scrutiny of the first test results and determination of the need for the subsequent test. When both tests are performed, the SPECT or PET MPI and coronary CTA images should be viewed independently and, using image fusion techniques, as an integrated image.

4. Fusion of radionuclide and CT images

Presently, fusion of radionuclide and CT images can be performed using images obtained from hybrid scanners (SPECT/CT or PET/CT) or using software to coregister images obtained on separate scanners. Fusion display of images typically includes fusion of CTAC and radionuclide images and fusion of CTA and radionuclide images. The CT images are typically displayed in gray scale, and the radionuclide images are displayed in a color scale. Software-based automated registration of the CT and emission images is accurate and rapid (in a few seconds) (116). The automated registration of CT and emission images is reviewed and manually adjusted as needed. Correct registration in 3-dimensional voxels is important to accurately localize the region of radiotracer uptake and to accurately generate attenuation maps. Automated coregistration of cardiac images is more challenging than that of other images because of cardiac motion, cardiac torsion, cardiac phases (gating), and breathing motion (117). The combination of MPI and CTA studies may improve the accuracy of each of the methods; CTA can improve MPI by better defining the contours of the myocardium, and MPI can aid in determining the significance of uninterpretable segments on CTA due to dense calcification, coronary artery motion, and other reasons (118).

5. CTAC and emission images

Attenuation correction is typically performed with CTAC obtained from hybrid scanners. Use of a CT scan obtained on a separate scanner for attenuation correction of the emission images is possible with software (119). However, the accuracy of this technique is not well studied and not widely used. Most vendors offer software to display the CTAC images along with the emission images in the 3 standard radiologic planes (transaxial, corona, and sagittal), and some vendors offer the ability to view these images in the standard cardiac imaging planes (short-axis, horizontal long-axis, and vertical long-axis images). It is recommended that registration be checked in multiple planes to ensure good registration.

If the images are misregistered, mere realignment of the CT and emission images using the fusion software is not adequate for correcting the errors in attenuation correction. The emission images need to be realigned to the transmission images and a new attenuation map generated (typically at the scanner acquisition console). The emission images then need to be reconstructed using the new attenuation map.

6. CTA and emission images

Radionuclide and coronary CTA imaging can be performed sequentially on a hybrid scanner or on separate dedicated SPECT, PET, and CT scanners (119). Although the CTAC and emission images are typically acquired sequentially as a part of the same study using hybrid devices, emission and CTA images are more commonly obtained as separate studies. The second test is performed if needed after a review of the results of the first study.

For fusion of the coronary CTA images, software programs are available that can segment the coronary CTA images and overlay them on the volume-rendered rest or stress myocardial perfusion images $(86,88)$ or coronary flow reserve images (quantitative PET images) (120). In clinical practice, the fusion images are typically used to map or localize the abnormal territory on emission images to the diseased vessel on coronary CTA (121). For research applications, hybrid imaging of radionuclide and CTA images is obtained for localization of radionuclide uptake $(122,123)$. The fused images can be helpful to localize diseased regions but should not be the sole images used for diagnostic interpretation. The respective MPI and CTA images should be reviewed for a diagnostic interpretation. Preliminary studies suggest that fused CTA and MPI images may help improve identification of obstructive disease, compared with separate review of CTA and MPI data (119).

Fusion of parametric images of coronary flow reserve with coronary CTA is possible $(120,124)$. A recent study demonstrated that CTA and quantitative ${ }^{15} \mathrm{O}$-water PET have high diagnostic accuracy in independently diagnosing flow limitation from $\mathrm{CAD}$, when compared with fractional flow reserve measurements by invasive coronary angiography (sensitivity, specificity, and accuracy of multidetectorrow $\mathrm{CT}$ and ${ }^{15} \mathrm{O}$-water PET was $95 \%, 87 \%, 90 \%$, and $95 \%$, $91 \%$, and $92 \%$, respectively). However, diagnostic accuracy increased significantly when the information was combined (sensitivity, specificity, and accuracy of hybrid imaging were $95 \%, 100 \%$, and $98 \%$, respectively). Also, fused images allowed identification of patients with stenosed vessels on CTA without flow limitations and identification of patients with microvascular dysfunction who did not have obstructive epicardial CAD.

\section{DOCUMENTATION/REPORTING}

The interpretation and reporting of the MPI studies is a complex topic that has been well described previously $(6,110,125-127)$. The coronary CTA report should include the indication for the study, imaging technique, description of findings, and limitations of the examination as previously suggested (Table 9) $(28,110,128)$. 
TABLE 9

Reporting of Coronary CTA Findings

\begin{tabular}{|c|c|}
\hline Report parameter & Details \\
\hline \multicolumn{2}{|l|}{ Indication for examination } \\
\hline \multirow[t]{6}{*}{ Imaging technique } & Scanner type (number of detectors, rotation time) \\
\hline & Acquisition type (gating method; tube voltage; dose modulation, if used; estimated radiation dose) \\
\hline & Administration of contrast agents (type, dose, route) \\
\hline & Medications used (vasodilator, nitroglycerin, or $\beta$-blocker) \\
\hline & Workstation methods for image reconstruction (slice thickness, phases of cardiac cycle) \\
\hline & Complications, if present \\
\hline \multirow[t]{10}{*}{ Description of findings } & Overall description of image quality/diagnostic confidence \\
\hline & Anomalies of coronary origin \\
\hline & Right- or left-dominant system \\
\hline & Location and size of any coronary artery aneurysm or dilatation \\
\hline & Description of atherosclerotic narrowing for vessels $\geq 2 \mathrm{~mm}$ in diameter (coronary CTA) \\
\hline & Location of atherosclerotic narrowing by anatomic landmarks \\
\hline & Diffuse or focal disease description \\
\hline & Description using 18-segment model of Society of Cardiovascular Computed Tomography \\
\hline & Noncardiac findings (e.g., adjacent lung fields, aorta) \\
\hline & Ventricular size and function when requested, if appropriate software available \\
\hline \multirow[t]{3}{*}{ Limitations of examination } & Heavy calcification (coronary CTA) \\
\hline & Motion abnormalities, arrhythmia \\
\hline & Difficulties with contrast injection \\
\hline Summary & Impression and recommendation \\
\hline
\end{tabular}

Further, for a comprehensive hybrid myocardial perfusion and cardiac CT report, the methods, findings, and interpretation of the MPI and coronary CT studies should be integrated (Table
10). The tables in this section are not as comprehensive as some of the prior publications on this topic but highlight some of the most important elements.

TABLE 10

Reporting of Hybrid Cardiac SPECT/CT and PET/CT Studies

\begin{tabular}{|c|c|}
\hline Report parameter & Details \\
\hline Indication for test & For both the MPI and the CT studies \\
\hline History & Prior coronary artery disease or coronary procedures \\
\hline Current medications & Listing of all \\
\hline Methods & Technique for the MPI study, stress study, and CT study \\
\hline MPI & $\begin{array}{l}\text { Type and dose of injected radiotracer; type of imaging technique (SPECT or PET); type of } \\
\text { attenuation correction, if performed; details of stress testing modality; hemodynamic and } \\
\text { electrocardiographic changes }\end{array}$ \\
\hline \multirow[t]{3}{*}{ CT } & $\begin{array}{l}\text { Technique for attenuation correction CT, coronary artery calcium score, and coronary CTA, } \\
\text { if applicable }\end{array}$ \\
\hline & Doses of $\beta$-blocker/nitroglycerin for coronary CTA, if used \\
\hline & Coronary CTA methods (Table 9) \\
\hline \multirow[t]{5}{*}{ Findings } & Nongated MPI findings \\
\hline & Gated MPI findings \\
\hline & $\begin{array}{l}\text { Coronary artery calcium score (overall Agatston score and percentile ranking based } \\
\text { on age and sex) }\end{array}$ \\
\hline & Coronary CTA details (Table 9) \\
\hline & $\begin{array}{l}\text { Ancillary findings (for MPI, CTAC, coronary artery calcium score, and coronary CTA, } \\
\text { if applicable) }\end{array}$ \\
\hline \multirow[t]{5}{*}{ Summary } & Impression of MPI findings \\
\hline & Impression of CT findings (especially for coronary artery calcium score and coronary CTA) \\
\hline & Integration of MPI and CT findings (especially for coronary artery calcium score and coronary CTA) \\
\hline & Explanations for discordant findings \\
\hline & Localization of disease to vascular territory \\
\hline
\end{tabular}


The methods section should incorporate not only the stress and MPI parameters but also the acquisition parameters for the cardiac CT scan (attenuation correction, coronary artery calcium scoring, and coronary CTA). The study findings of MPI, coronary artery calcium scoring, coronary CTA, and ancillary findings should be reported in distinct sections. MPI findings should be reported, including the elements listed in Table 10 and as suggested previously. CT findings should be reported, including the elements listed in Tables 9 and 10 and as suggested previously, using standard terminology (60). The final-interpretation section must include both the MPI results and the coronary artery calcium score or coronary CTA results when applicable in an integrated comprehensive statement.

Incidental findings are frequent (129) in the low-dose CTAC scans and coronary CTA studies. Although most such findings are of no relevance, clinically important findings that may affect patient management and are otherwise unknown are detected in up to $5 \%$ of patients (130). However, there is presently no consensus on reporting findings seen incidentally on cardiac CT studies. Some guidelines recommend that the CTAC, coronary artery calcium score, and coronary CTA studies be reviewed and reported by an experienced physician with appropriate training in chest CT interpretation (13), whereas others report no consensus on this matter (29). It is the consensus of this writing group that the CT scans from hybrid SPECT or PET be reviewed and reported for ancillary findings.

\section{EQUIPMENT SPECIFICATION}

The image processing and display for the MPI images from hybrid devices are similar to those for stand-alone scanners and should be performed using previously described standards $(5,6)$. Dedicated workstations with cardiac display software for MPI should be used. For PET MPI, only attenuation-corrected scans should be interpreted. With SPECT, both noncorrected and attenuation-corrected images should be reviewed when both are acquired. Dedicated software should be available to review the registration of the myocardial perfusion and CTAC images. Dedicated workstations should be used to compute the coronary artery calcium score and to review the coronary CTA studies. It is important to review the axial source images and multiplanar reformatted coronary CTA images, as well as maximum-intensity images, when doing such would be useful. Volume-rendered and fusion images may be helpful for disease localization but should not be used for primary diagnostic purposes. Several vendors offer unified platforms for the review and interpretation of myocardial perfusion, coronary artery calcium score, and coronary CTA images.

\section{QUALITY CONTROL (QC) AND IMPROVEMENT, SAFETY, INFECTION CONTROL, AND PATIENT EDUCATION CONCERNS}

\section{A. Hardware QC}

The standard QC steps for SPECT, PET, and CT scanners apply to hybrid devices (5). Separate QC procedures are required for the CT scanner and the SPECT or PET scanner in a hybrid device. The QC procedures for SPECT and PET scanners minimally include daily, weekly, monthly, and quarterly camera QC as per manufacturer recommendations. In addition, the requirements of accrediting organizations, that is, the ACR and the Intersocietal Commission for the Accreditation of Nuclear Medicine
Laboratories, must be met for an accredited facility. This means that more frequent $\mathrm{QC}$ and testing will be required. To specify in this document what should be done will be difficult since each manufacturer requires slightly different tests at different frequencies.

SPECT scanners need daily energy peaking, uniformity testing, and weekly resolution and linearity testing as described previously (5). Center-of-rotation and uniformity corrections are recommended depending on the specific scanner manufacturer guidelines.

The QC procedures for PET scanners have been previously described (6). PET scanners need QC testing at the time of delivery and after any major upgrades. Daily testing of the PET system is as recommended by the manufacturer. Detector gains and coincidence-timing checks or adjustments should be performed at least weekly. Detector normalization should be performed at least quarterly, and PET scanners should also be calibrated or tested for absolute-activitymeasurement accuracy and overall system performance (including spatial registration between the PET and CT images) at least quarterly. It is also recommended that the accuracy of attenuation and scatter compensation be evaluated at least annually.

CT scanners need to be checked for CT number calibration and field uniformity, with periodic checks of spatial resolution (131).

\section{B. Imaging QC}

1. MPI

MPI images are checked routinely for several QC steps (5). Raw projection SPECT images are reviewed for patient motion, attenuation patterns, extracardiac activity, subdiaphragmatic activity, and position of the arms. Reconstructed SPECT and PET images are reviewed for count density, appropriate alignment between the stress and rest images, hot spots, and contours for calculation of transient cavity dilation ratio. Gated images are reviewed for count density, the beat histogram is checked for arrhythmias, and the contours of the gated files are checked to measure the accuracy of left ventricular volumes.

2. CT

CT images are checked for motion artifacts and for hardware and software artifacts. Coronary artery calcium score and coronary CTA images need to be reviewed for motion artifacts $(13,28,29)$. Multiple datasets in different cardiac phases may need to be reconstructed until the dataset with fewest artifacts is identified.

3. Imaging QC unique to hybrid devices

Misregistration of emission and transmission images can result in artifacts (132-134). Therefore, registration of the CT and MPI images must be routinely verified before image reconstruction and interpretation. This QC also involves ensuring that the MPI image is of adequate quality (count density and without excessive motion) and that the CT images are of adequate quality. If the transmission and emission images are determined to be of adequate quality, then the registration of these images is checked.

4. Fusion software

Several commercial software programs are available for fusion of CT and MPI images acquired from the same 
scanner or separate scanners. A fusion software package can also be provided by the hardware vendors. The results of the fusion should always be checked for accuracy before image interpretations.

5. Sources of error

Common sources of error for SPECT (5) and PET (6) radionuclide imaging are patient motion, poor signal-tonoise ratio, attenuation artifacts, and reconstruction artifacts. In addition, the primary sources of error in hybrid imaging are related to artifacts from misregistration of the transmission and emission scans and from motion during CTAC, coronary artery calcium scoring, or coronary CTA.

Misregistration is the most common source of artifacts on PET or SPECT/CT. This can result from several factors. Patient motion can occur between the transmission and emission images. In addition, the position of the heart on MPI relative to that on CT can change because of the effects of vasodilator stress, or because imaging for CTAC occurs during a phase of the respiratory cycle that results in the myocardium not being positioned at the average heart position, as is represented by the PET image.

Software reregistration may be used to correct for misregistration of the transmission and emission images when the source emission and transmission images are motionfree and of good quality. However, if either transmission or emission images are degraded by motion, software solutions may not be able to adequately correct the degradation and the scan may have to be repeated.

Patients should be instructed not to move, and the operators should make note of involuntary movements (e.g., coughing or sneezing during image acquisition). Patient motion during PET MPI is difficult to discern, and motion during either the MPI or the CT scan limits the use of those data. The MPI scan can be reacquired with SPECT radiotracers. With PET, the dynamic image file can be unlisted, the frames with motion discarded, and the remaining frames summed; however, this option may not be optimal with short-half-life radiotracers (especially if motion occurs during the initial 1-3 min of an ${ }^{82} \mathrm{Rb}$ image acquisition). The 75 -s half-life of ${ }^{82} \mathrm{Rb}$

\section{TABLE 11}

Possible Approaches to Reduce Radiation Dose from MPI, CT, and Hybrid Procedures

\begin{tabular}{|c|c|c|}
\hline Procedure & Approach & Notes \\
\hline \multirow[t]{6}{*}{ MPI (136) } & Weight-based radiotracer use & Smaller subjects may receive lower radiotracer dose. \\
\hline & Single-isotope protocols & $\begin{array}{l}\text { Dual-isotope imaging with }{ }^{201} \mathrm{TI} \text { rest and }{ }^{99 \mathrm{mT}} \mathrm{Tc} \text { stress protocols } \\
\text { have higher dose estimates than single-isotope protocols. }\end{array}$ \\
\hline & Lower-dose radiotracer & $\begin{array}{l}\text { High sensitivity of newer scanners with solid-state detectors } \\
\text { allows for rapid scanning or lower-dose injection, reducing } \\
\text { estimated dose by almost a third compared with conventional } \\
\text { SPECT (139); new software reconstructions }(140-142,152) \text { allow } \\
\text { for half-time or quarter-time image acquisition, which can be } \\
\text { used to perform reduced-dose imaging at full-time acquisition. }\end{array}$ \\
\hline & Stress-only MPI* & Rest imaging can be avoided, and throughput is faster. \\
\hline & PET MPI & $\begin{array}{l}\text { PET perfusion radiotracers }\left({ }^{13} \mathrm{~N} \text {-ammonia and }{ }^{82} \mathrm{Rb}\right) \text { result in lower } \\
\text { effective radiation dose than do SPECT radiotracers (Table } 12) .\end{array}$ \\
\hline & & $\begin{array}{l}\text { Compared with 2-dimensional PET, 3-dimensional imaging uses } \\
\text { lower radiotracer dose and consequently has lower radiation } \\
\text { dose to patients. }\end{array}$ \\
\hline \multirow[t]{4}{*}{ CT (144) } & $\begin{array}{l}\text { Prospective ECG-triggered } \\
\text { imaging }\end{array}$ & $\begin{array}{l}\text { When available, prospective ECG-triggered imaging or high-pitch } \\
\text { acquisition is preferred over retrospective gating to reduce dose } \\
\text { (63); reduce padding to minimize dose (64). }\end{array}$ \\
\hline & ECG dose modulation & $\begin{array}{l}\text { Reducing tube current during systolic phases reduces dose by } \\
\sim 25 \%-40 \% \text {, depending on heart rate and settings }(143,144) \text {. }\end{array}$ \\
\hline & Low-kV imaging & $\begin{array}{l}\text { There is } 53 \% \text { reduction in median dose estimates for } 100-k V \text { scan, } \\
\text { compared with } 120 \mathrm{kV} \text { scan (144); this is particularly helpful in } \\
\text { patients with low body mass index or for specific indications (65). }\end{array}$ \\
\hline & $\begin{array}{l}\text { Low-mA imaging with iterative } \\
\text { reconstruction algorithms }\end{array}$ & $\begin{array}{l}\text { Technique uses low tube current combined with adaptive statistical } \\
\text { iterative reconstruction (instead of filtered backprojection) of images } \\
\text { to minimize image noise level and maintain image quality (66). }\end{array}$ \\
\hline $\begin{array}{l}\text { SPECT/CTA and } \\
\text { PET/CTA }\end{array}$ & Personalized protocol & $\begin{array}{l}\text { For example, use of prospective ECG-triggered axial coronary CTA } \\
\text { and low-dose stress-only cardiac SPECT ( } 97 \text { ) or PET may reduce } \\
\text { collective radiation dose by approximately } 40 \% \text {. }\end{array}$ \\
\hline
\end{tabular}

*Stress-only imaging can be considered in highly selected cohorts with very low likelihood of coronary artery disease and when attenuation correction is available.

$\mathrm{CTA}=\mathrm{CT}$ angiogram; MPI = myocardial perfusion imaging. 
allows for repeating the study if excessive motion or other uncorrectable artifacts are observed.

Patient motion during a CT scan may cause enough streak artifacts to require a repeated CT scan. Coronary artery motion can be minimized for dated cardiac CT scans (coronary artery calcium scoring and coronary CTA) using $\beta$-blockers as needed to reduce the patient heart rate at the time of image acquisition. However, $\beta$-blockers are generally not used with coronary artery calcium scoring. Additionally, it has been shown that the extent and severity of MPI abnormalities are reduced in patients under the effects of $\beta$-blockers $(31,32)$. Stair-step artifacts are eliminated on 320-slice scanners wherein the entire heart volume is covered in

TABLE 12

Radiation Dosimetry: Adults*

\begin{tabular}{|c|c|c|c|c|c|c|c|c|}
\hline \multirow[b]{2}{*}{ Radiopharmaceutical } & \multicolumn{3}{|c|}{ Largest radiation dose } & \multicolumn{2}{|c|}{ Effective dose } & \multicolumn{2}{|c|}{$\begin{array}{l}\text { Administered } \\
\text { activity }\end{array}$} & \multirow{2}{*}{$\begin{array}{c}\text { Relative } \\
\text { radiation level }\end{array}$} \\
\hline & Organ & mGy/MBq & $\mathrm{rad} / \mathrm{mCi}$ & $\mathrm{mSv} / \mathrm{MBq}$ & $\mathrm{rem} / \mathrm{mCi}$ & $\mathrm{MBq}$ & $\mathrm{mCi}$ & \\
\hline 99mTc-sestamibi, rest ${ }^{\star}$ & Gallbladder & 0.039 & 0.14 & 0.009 & 0.033 & $296-444$ & $8-12$ & 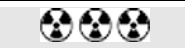 \\
\hline 99mTc-sestamibi, stress* & Gallbladder & 0.033 & 0.12 & 0.0079 & 0.029 & $888-1,332$ & $24-36$ & 383 \\
\hline${ }^{99 \mathrm{~m} T c-t e t r o f o s m i n}$, rest $^{\dagger}$ & Gallbladder & 0.027 & 0.10 & 0.0069 & 0.026 & $296-444$ & 8-12 & $(3)$ \\
\hline${ }^{99 m}$ Tc-tetrofosmin, exercise ${ }^{\dagger}$ & Gallbladder & 0.027 & 0.10 & 0.0069 & 0.026 & $888-1,332$ & $24-36$ & $(3)$ \\
\hline${ }^{201} \mathrm{TI}^{\dagger}$ & Kidneys & 0.48 & 1.8 & 0.14 & 0.52 & 111-185 & $3-5$ & $(3)(3)$ \\
\hline${ }^{82} \mathrm{Rb}^{\ddagger}$ & Kidneys & 0.0093 & 0.034 & 0.0017 & 0.0063 & $370-2,220$ & $10-60$ & 88 \\
\hline${ }^{13} \mathrm{~N}-$ ammonia $^{\S}$ & Urinary bladder & 0.0081 & 0.030 & 0.0027 & 0.010 & $370-925$ & $10-25$ & 88 \\
\hline${ }^{18} \mathrm{~F}-\mathrm{FDG}^{\dagger}$ & Urinary bladder & 0.13 & 0.48 & 0.019 & 0.070 & $185-555$ & $5-15$ & 88 \\
\hline Scout CT & - & & & - & & - & & 3 \\
\hline CTAC & - & & & - & & - & & $(3)$ \\
\hline Coronary artery calcium score & - & & & - & & - & & 83 \\
\hline Coronary CTA & - & & & - & & - & & 88 \\
\hline 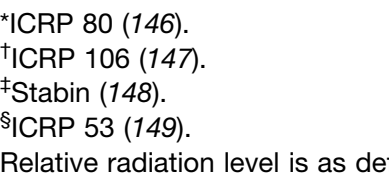 & d by ACR: & $=0.1$ & $\mathrm{v} ;$ & $=1$ & 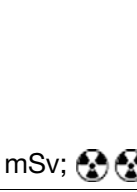 & 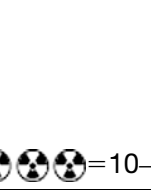 & $\mathrm{m}$ & \\
\hline
\end{tabular}

TABLE 13

Radiation Dosimetry in Children (5 Years old)

\begin{tabular}{|c|c|c|c|c|c|}
\hline \multirow[b]{2}{*}{ Radiopharmaceutical } & \multicolumn{3}{|c|}{ Largest radiation dose } & \multicolumn{2}{|c|}{ Effective dose } \\
\hline & Organ & mGy/MBq & $\mathrm{rad} / \mathrm{mCi}$ & $\mathrm{mSv} / \mathrm{MBq}$ & $\mathrm{rem} / \mathrm{mCi}$ \\
\hline 99mTc-sestamibi, rest* & Gallbladder & 0.10 & 0.37 & 0.028 & 0.10 \\
\hline 99mTc-sestamibi, stress* & Gallbladder & 0.086 & 0.32 & 0.023 & 0.085 \\
\hline 99mTc-tetrofosmin, rest $^{\dagger}$ & Gallbladder & 0.073 & 0.27 & 0.021 & 0.078 \\
\hline${ }_{99 m} T_{c}$-tetrofosmin, exercise ${ }^{\dagger}$ & Gallbladder & 0.073 & 0.27 & 0.021 & 0.078 \\
\hline${ }^{201} \mathrm{Tl}^{\dagger}$ & Testes & 3.6 & 13 & 0.79 & 2.9 \\
\hline${ }^{82} \mathrm{Rb}^{\ddagger}$ & Thyroid & 0.050 & 0.18 & 0.0063 & 0.023 \\
\hline${ }^{13} \mathrm{~N}$-ammonia & Urinary bladder & 0.024 & 0.089 & 0.0077 & 0.028 \\
\hline${ }^{18} \mathrm{~F}-\mathrm{FDG}{ }^{\dagger}$ & Urinary bladder & 0.34 & 1.3 & 0.056 & 0.21 \\
\hline \multicolumn{6}{|l|}{$\begin{array}{l}\text { †ICRP 106, page } 218 \text { (147). } \\
{ }^{\ddagger} \text { Stabin (148). }\end{array}$} \\
\hline Estimated dose (mSv) can be & $d$ as effective $a$ & nistered & & & \\
\hline
\end{tabular}


1-2 heartbeats $(13,28)$, as well as with the prospectively triggered high-pitch acquisition (FLASH mode [fast-pitch spiral CT]), which requires a fraction of 1 heart beat (approximately $250 \mathrm{~ms}$ ) for the CTA acquisition (135).

\section{RADIATION SAFETY AND ALARA ISSUES}

Patient exposure to ionizing radiation should be at the minimum dose consistent with obtaining a diagnostic examination in accordance with ALARA (as low as reasonably achievable) principles. Patient radiation exposure may be reduced by administering less radiopharmaceutical when the technique or equipment used for imaging can support such an action. Each patient procedure is unique, and the methodology to achieve minimum exposure while maintaining diagnostic accuracy needs to be viewed in this light. Dose ranges outlined in this document should be considered a guide, and it is expected that, at a minimum, dose reduction techniques will be considered for each patient procedure and used when appropriate (Tables 3, 11, and 12). Dose reduction is important not only for patients but also for the technologists and health professionals who perform these procedures. The lowest radiation dose required to obtain a diagnostic-quality image should be used.

The 2 options for hybrid imaging include dose reduction from CT studies or MPI procedures. Several factors influence radiation dose, and hence dose estimates are variable and their precise estimation is challenging $(136,137)$. Possible approaches to reduce radiation dose from MPI $(138), \mathrm{CT}(28,29)$, and hybrid procedures are shown in Table 11 (139-145).

\section{A. MPI Dosimetry}

For MPI dosimetry data, see Tables 12-19 (146-151)

\section{B. MPI in the Pregnant or Potentially Pregnant Patient}

Hybrid imaging is not recommended in the pregnant or the potentially pregnant patient, for whom imaging modalities without radiation risk are instead recommended.

\section{MPI in the Breast-Feeding Patient}

Radionuclide imaging is not recommended for the breast-feeding patient. Alternate imaging modalities with-

TABLE 14

Radiation Dosimetry in Fetus/Embryo: ${ }^{99 m T c-S e s t a m i b i ~}$

\begin{tabular}{llll}
\hline \multirow{2}{*}{$\begin{array}{c}\text { 99mTc-sestamibi } \\
\text { study }\end{array}$} & & \multicolumn{2}{c}{ Fetal dose } \\
\cline { 3 - 4 } Rest & Stage of gestation & mGy/MBq & rad/mCi \\
& Early & 0.015 & 0.055 \\
& $3 \mathrm{mo}$ & 0.012 & 0.044 \\
Stress & $6 \mathrm{mo}$ & 0.0084 & 0.031 \\
& $9 \mathrm{mo}$ & 0.0054 & 0.020 \\
& Early & 0.012 & 0.044 \\
& $3 \mathrm{mo}$ & 0.0095 & 0.035 \\
& $6 \mathrm{mo}$ & 0.0069 & 0.026 \\
& $9 \mathrm{mo}$ & 0.0044 & 0.016
\end{tabular}

Data are from Russell et al. (150). No information about possible placental crossover of this compound was available for use in estimating fetal doses.
TABLE 15

Radiation Dosimetry in Fetus/Embryo: ${ }^{99} \mathrm{Tc}-$ Tetrofosmin

\begin{tabular}{lcc}
\hline & \multicolumn{2}{c}{ Fetal dose } \\
\cline { 2 - 3 } Stage of gestation & $\mathrm{mGy} / \mathrm{MBq}$ & $\mathrm{rad} / \mathrm{mCi}$ \\
\hline Early & 0.0096 & 0.036 \\
$3 \mathrm{mo}$ & 0.0070 & 0.026 \\
$6 \mathrm{mo}$ & 0.0054 & 0.020 \\
$9 \mathrm{mo}$ & 0.0036 & 0.013
\end{tabular}

Data are from Russell et al. (150). No information about possible placental crossover of this compound was available for use in estimating fetal doses. Separate estimates were not given for rest and exercise subjects.

TABLE 16

Radiation Dosimetry in Fetus/Embryo: ${ }^{201} \mathrm{TI}$

\begin{tabular}{lcc}
\hline & \multicolumn{2}{c}{ Fetal dose } \\
\cline { 2 - 3 } Stage of gestation & $\mathrm{mGy} / \mathrm{MBq}$ & $\mathrm{rad} / \mathrm{mCi}$ \\
\hline Early & 0.097 & 0.36 \\
$3 \mathrm{mo}$ & 0.058 & 0.21 \\
$6 \mathrm{mo}$ & 0.047 & 0.17 \\
$9 \mathrm{mo}$ & 0.027 & 0.10
\end{tabular}

Data are from Russell et al. (150). No information about possible placental crossover of this compound was available for use in estimating fetal doses.

TABLE 17

Radiation Dosimetry in Fetus/Embryo: ${ }^{82} \mathrm{Rb}$

\begin{tabular}{lcl}
\hline & \multicolumn{2}{c}{ Fetal dose } \\
\cline { 2 - 3 } Stage of gestation & $\mathrm{mGy} / \mathrm{MBq}$ & $\mathrm{rad} / \mathrm{mCi}$ \\
\hline Early & 0.00028 & 0.0010 \\
$3 \mathrm{mo}$ & 0.00025 & 0.00092 \\
$6 \mathrm{mo}$ & 0.00023 & 0.00085 \\
$9 \mathrm{mo}$ & 0.00021 & 0.00078
\end{tabular}

Data are from Russell et al. (150) but were estimated from the model of Stabin (148). No information about possible placental crossover of this compound was available for use in estimating fetal doses.

out radiation risk are instead recommended. If radionuclide imaging is considered, the International Commission on Radiation Protection publication 106 (147) suggests a 48-h interruption of breast feeding for subjects receiving ${ }^{201} \mathrm{Tl}$ but does not provide a recommendation about interruption of breast feeding for ${ }^{82} \mathrm{Rb}$. The authors recommend that no interruption is needed for breast-feeding patients administered ${ }^{99 \mathrm{~m}} \mathrm{Tc}$ agents, ${ }^{13} \mathrm{~N}$-ammonia, or ${ }^{18} \mathrm{~F}$-FDG. Close and prolonged contact $(>2 \mathrm{~min}$ ) should be avoided for a few hours after ${ }^{18} \mathrm{~F}$-FDG administration. However, for ${ }^{99 \mathrm{~m}} \mathrm{Tc}$ radiopharmaceuticals, consideration should be given to postponing the procedure in a lactating woman. A 4-h in- 
TABLE 18

Radiation Dosimetry in Fetus/Embryo: ${ }^{13} \mathrm{~N}-A m m o n i a$

\begin{tabular}{lcc}
\hline & \multicolumn{2}{c}{ Fetal dose } \\
\cline { 2 - 3 } Stage of gestation & $\mathrm{mGy} / \mathrm{MBq}$ & $\mathrm{rad} / \mathrm{mCi}$ \\
\hline Early & 0.0023 & 0.0085 \\
$3 \mathrm{mo}$ & 0.0020 & 0.0074 \\
$6 \mathrm{mo}$ & 0.0016 & 0.0059 \\
$9 \mathrm{mo}$ & 0.0015 & 0.0056
\end{tabular}

No dose estimates to fetus were provided by Russell et al. (150), but estimates were made using kinetic data in ICRP 53 (149). No information about possible placental crossover of this compound was available for use in estimating fetal doses.

TABLE 19

Radiation Dosimetry in Fetus/Embryo: ${ }^{18} \mathrm{~F}-\mathrm{FDG}$

\begin{tabular}{lcc}
\hline & \multicolumn{2}{c}{ Fetal dose } \\
\cline { 2 - 3 } Stage of gestation & $\mathrm{mGy} / \mathrm{MBq}$ & $\mathrm{rad} / \mathrm{mCi}$ \\
\hline Early & 0.022 & 0.081 \\
$3 \mathrm{mo}$ & 0.022 & 0.081 \\
$6 \mathrm{mo}$ & 0.017 & 0.063 \\
$9 \mathrm{mo}$ & 0.017 & 0.063
\end{tabular}

Dose estimates were taken from Stabin (151). Information about placental crossover was included in the model.

terruption during which 1 meal is discarded can be considered to be on the safe side.

\section{Coronary CTA}

Coronary CTA can now be performed with effective doses of less than $1 \mathrm{mSv}$ in some patients. The typical radiation doses from cardiac CT are listed in Table 12.

\section{CONCLUSION}

Advances in hybrid imaging technologies over the past several years have resulted in the ability to improve image quality and the diagnostic accuracy of MPI and identify calcified atherosclerosis (coronary artery calcium scoring) and coronary artery stenoses noninvasively using coronary CTA. For successful and appropriate use of this new technology, a clear understanding of the capabilities and limitations of the technology and appropriate patient selection, preparation, scan acquisition, and image reconstruction is required. This document attempts to provide some guidance on the performance of hybrid cardiac procedures using PET/CT and SPECT/CT. For more details, readers are referred to other documents detailing the protocols and procedures for each of these tests. The proper performance of hybrid imaging requires clinical knowledge and appropriate training and expertise at all steps of the process from screening to the performance, interpretation, and reporting of these procedures, so that each procedure may be tailored to the patient and the clinical question to be answered.

\section{REFERENCES}

1. Klocke FJ, Baird MG, Lorell BH, et al. ACC/AHA/ASNC guidelines for the clinical use of cardiac radionuclide imaging: executive summary-a report of the American College of Cardiology/American Heart Association Task Force on Practice Guidelines (ACC/AHA/ASNC Committee to Revise the 1995 Guidelines for the Clinical Use of Cardiac Radionuclide Imaging). J Am Coll Cardiol. 2003;42:1318-1333.

2. Procedure guideline for myocardial perfusion imaging 3.3. Society of Nuclear Medicine and Molecular Imaging Web site. Available at: http://interactive. snmmi.org/docs/155.pdf. Published September 2008. Accessed March 1, 2012.

3. Practice Guideline for the Performance of Cardiac Scintigraphy. American College of Radiology Web site. Available at: http://www.acr.org/Secondary MainMenuCategories/quality_safety/guidelines/nuc_med/cardiac_scintigraphy. aspx. Published 2009. Accessed March 1, 2012

4. Hendel RC, Berman DS, Di Carli MF, et al. ACCF/ASNC/ACR/AHA/ASE/ SCCT/SCMR/SNMMI 2009 Appropriate use criteria for cardiac radionuclide imaging: a report of the American College of Cardiology Foundation Appropriate Use Criteria Task Force, the American Society of Nuclear Cardiology, the American College of Radiology, the American Heart Association, the American Society of Echocardiography, the Society of Cardiovascular Computed Tomography, the Society for Cardiovascular Magnetic Resonance, and the Society of Nuclear Medicine and Molecular Imaging_endorsed by the American College of Emergency Physicians. J Am Coll Cardiol. 2009;53:2201-2229.

5. Holly TA, Abbott BG, Al-Mallah M, et al. Single photon-emission computed tomography. American Society of Nuclear Cardiology Web site. Available at: http://www.asnc.org/imageuploads/ImagingGuidelineSPECTJune2010.pdf. Published June 15, 2010. Accessed March 1, 2012.

6. Dilsizian V, Bacharach SL, Beanlands RS, et al. PET myocardial perfusion and metabolism clinical imaging. American Society of Nuclear Cardiology Web site. Available at: http://www.asnc.org/imageuploads/ImagingGuidelinesPETJuly2009.pdf. Published 2008. Accessed March 12, 2012.

7. Di Carli MF. Hybrid imaging: integration of nuclear imaging and cardiac CT. Cardiol Clin. 2009;27:257-263.

8. Bengel FM, Higuchi T, Javadi MS, Lautamaki R. Cardiac positron emission tomography. J Am Coll Cardiol. 2009;54:1-15.

9. Buck AK, Nekolla S, Ziegler S, et al. SPECT/CT. J Nucl Med. 2008;49:13051319.

10. Bateman TM, Heller GV, McGhie AI, et al. Diagnostic accuracy of rest/stress ECG-gated Rb-82 myocardial perfusion PET: comparison with ECG-gated Tc99m sestamibi SPECT. J Nucl Cardiol. 2006;13:24-33.

11. Flotats A, Knuuti J, Gutberlet M, et al. Hybrid cardiac imaging: SPECT/CT and PET/CT - a joint position statement by the European Association of Nuclear Medicine (EANM), the European Society of Cardiac Radiology (ESCR) and the European Council of Nuclear Cardiology (ECNC). Eur J Nucl Med Mol Imaging. 2011;38:201-212.

12. Taylor AJ, Cerqueira M, Hodgson JM, et al. ACCF/SCCT/ACR/AHA/ASE/ ASNC/NASCI/SCAI/SCMR 2010 appropriate use criteria for cardiac computed tomography: a report of the American College of Cardiology Foundation Appropriate Use Criteria Task Force, the Society of Cardiovascular Computed Tomography, the American College of Radiology, the American Heart Association, the American Society of Echocardiography, the American Society of Nuclear Cardiology, the North American Society for Cardiovascular Imaging, the Society for Cardiovascular Angiography and Interventions, and the Society for Cardiovascular Magnetic Resonance. J Am Coll Cardiol. 2010;56:18641894.

13. ACR practice guideline for the performance and interpretation of cardiac computed tomography. American College of Radiology Web site. Available at: http://www.acr.org/SecondaryMainMenuCategories/quality_safety/guidelines/ dx/cardio/ct_cardiac.aspx. Published 2011. Accessed March 1, 2012.

14. Conjoint statement of the SNMMI and the ACNP on credentialing and delineation of privileges for cardiac PET. J Nucl Med. 2007;5:850-851.

15. Rodgers GP, Ayanian JZ, Balady G, et al. American College of Cardiology/ American Heart Association clinical competence statement on stress testing: a report of the American College of Cardiology/American Heart Association/ American College of Physicians-American Society of Internal Medicine Task Force on Clinical Competence. Circulation. 2000;102:1726-1738.

16. The SNMMI procedure guideline for general imaging 6.0. Society of Nuclear Medicine and Molecular Imaging Web site. Available at: http://interactive. snmmi.org/index.cfm?pageID=772. Published 2010. Accessed March 13, 2012.

17. Cerqueira MD, Berman DS, Di Carli MF, Schelbert HR, Wackers FJ, Williams KA. Task force 5: training in nuclear cardiology endorsed by the American Society of Nuclear Cardiology. J Am Coll Cardiol. 2008;51:368-374.

18. ACR/SNMMI technical standard for diagnostic procedures using radiopharmaceuticals. American College of Radiology Web site. Available at: http://www. acr.org/SecondaryMainMenuCategories/quality_safety/guidelines/nuc_med/ radiopharmaceuticals.aspx. Published 2011. Accessed March 1, 2012.

19. ACGME program requirements for graduate medical education in nuclear medicine. Accreditation Council for Graduate Medical Education Web site. 
Available at: http://www.acgme.org/acWebsite/downloads/RRC_progReq/200_ nuclear_medicine_07012007.pdf. Published July 1, 2007. Accessed November 19, 2010, 2010.

20. Conjoint statement of the SNMMI and the ACNP on credentialing and delineation of privileges for cardiovascular CT. J Nucl Med. 2007;5:852-853.

21. Budoff MJ, Achenbach S, Berman DS, et al. Task force 13: training in advanced cardiovascular imaging (computed tomography) endorsed by the American Society of Nuclear Cardiology, Society of Atherosclerosis Imaging and Prevention, Society for Cardiovascular Angiography and Interventions, and Society of Cardiovascular Computed Tomography. J Am Coll Cardiol. 2008;51:409-414.

22. Budoff MJ, Cohen MC, Garcia MJ, et al. ACCF/AHA clinical competence statement on cardiac imaging with computed tomography and magnetic resonance: a report of the American College of Cardiology Foundation/American Heart Association/American College of Physicians Task Force on Clinical Competence and Training. J Am Coll Cardiol. 2005;46:383-402.

23. Scope of practice for the nuclean medicine technologist. Society of Nuclear Medicine and Molecular Imaging Web site. Available at: http://interactive. snmmi.org/index.cfm?PageID=2637. Accessed March 27, 2012.

24. Delbeke D, Coleman RE, Guiberteau MJ, et al. Procedure guideline for SPECT/ CT imaging 1.0. J Nucl Med. 2006;47:1227-1234.

25. Delbeke D, Coleman RE, Guiberteau MJ, et al. Procedure guideline for tumor imaging with ${ }^{18}$ F-FDG PET/CT 1.0. J Nucl Med. 2006;47:885-895.

26. Suggested State Regulations for Control of Radiation Part A: Medical Credentialing. Frankfort, KY: The Conference of Radiation Control Program Directors. February 2012. Z1-Z19.

27. Advanced Diagnostic Imaging Accreditation. CMS.gov Web site. Available at: https://www.cms.gov/MedicareProviderSupEnroll/03_AdvancedDiagnosticImagingAccreditation.asp. Accessed March 1, 2012.

28. Abbara S, Arbab-Zadeh A, Callister TQ, et al. SCCT guidelines for performance of coronary computed tomographic angiography: a report of the Society of Cardiovascular Computed Tomography Guidelines Committee. J Cardiovasc Comput Tomogr. 2009;3:190-204.

29. Mark DB, Berman DS, Budoff MJ, et al. ACCF/ACR/AHA/NASCI/SAIP/SCAI/ SCCT 2010 expert consensus document on coronary computed tomographic angiography: a report of the American College of Cardiology Foundation Task Force on Expert Consensus Documents. Circulation. 2010;121:2509-2543.

30. Greenberger P, Patterson R. Prednisone-diphenhydramine regimen prior to use of radiographic contrast media. J Allergy Clin Immunol. 1979;63:295.

31. Yoon AJ, Melduni RM, Duncan SA, Ostfeld RJ, Travin MI. The effect of betablockers on the diagnostic accuracy of vasodilator pharmacologic SPECT myocardial perfusion imaging. J Nucl Cardiol. 2009;16:358-367.

32. Reyes E, Stirrup J, Roughton M, D'Souza S, Underwood SR, Anagnostopoulos C. Attenuation of adenosine-induced myocardial perfusion heterogeneity by atenolol and other cardioselective beta-adrenoceptor blockers: a crossover myocardial perfusion imaging study. J Nucl Med. 2010;51:1036-1043.

33. Kloner RA. Pharmacology and drug interaction effects of the phosphodiesterase 5 inhibitors: focus on alpha-blocker interactions. Am J Cardiol. 2005;96:42M$46 \mathrm{M}$.

34. Chang SM, Nabi F, Xu J, Raza U, Mahmarian JJ. Normal stress-only versus standard stress/rest myocardial perfusion imaging: similar patient mortality with reduced radiation exposure. J Am Coll Cardiol. 2010;55:221-230.

35. Duvall WL, Wijetunga MN, Klein TM, et al. The prognosis of a normal stress-only Tc-99m myocardial perfusion imaging study. J Nucl Cardiol. 2010;17:370-377.

36. Des Prez RD, Dahlberg ST, Einstein AJ, et al. ASNC Announcement. J Nucl Cardiol. 2009;16:329.

37. Yamagishi H, Shirai N, Takagi M, et al. Identification of cardiac sarcoidosis with ${ }^{13} \mathrm{~N}-\mathrm{NH}_{3} /{ }^{18}$ F-FDG PET. J Nucl Med. 2003;44:1030-1036.

38. Okumura W, Iwasaki T, Toyama T, et al. Usefulness of fasting ${ }^{18} \mathrm{~F}-\mathrm{FDG}$ PET in identification of cardiac sarcoidosis. J Nucl Med. 2004;45:1989-1998.

39. Youssef G, Leung E, Mylonas I, et al. The use of ${ }^{18} \mathrm{~F}$-FDG PET in the diagnosis of cardiac sarcoidosis: a systematic review and metaanalysis including the Ontario experience. J Nucl Med. 2012;53:241-248.

40. Ohira H, Tsujino I, Yoshinaga K. ${ }^{18} \mathrm{~F}$-fluoro-2-deoxyglucose positron emission tomography in cardiac sarcoidosis. Eur J Nucl Med Mol Imaging. 2011;38: 1773-1783.

41. Williams G, Kolodny GM. Suppression of myocardial ${ }^{18}$ F-FDG uptake by preparing patients with a high-fat, low-carbohydrate diet. AJR. 2008;190:W151W156.

42. Conjoint statement of the SNMMI and the ACNP on credentialing and delineation of privileges for body PET. J Nucl Med. 2006;47:1559-1560.

43. Conjoint statement of the SNMMI and the ACNP on credentialing and delineation of privileges for CT performed in conjunction with body SPECT or PET. J Nucl Med. 2006;47:1561-1562.
44. Masood Y, Liu YH, Depuey G, et al. Clinical validation of SPECT attenuation correction using X-ray computed tomography-derived attenuation maps: multicenter clinical trial with angiographic correlation. J Nucl Cardiol. 2005;12:676-686.

45. Bacharach SL. PET/CT attenuation correction: breathing lessons. J Nucl Med. 2007;48:677-679.

46. Koepfli P, Hany TF, Wyss CA, et al. CT attenuation correction for myocardial perfusion quantification using a PET/CT hybrid scanner. J Nucl Med. 2004;45: $537-542$.

47. Souvatzoglou M, Bengel F, Busch R, et al. Attenuation correction in cardiac PET/CT with three different CT protocols: a comparison with conventional PET. Eur J Nucl Med Mol Imaging. 2007;34:1991-2000.

48. Fricke E, Fricke H, Weise R, et al. Attenuation correction of myocardial SPECT perfusion images with low-dose CT: evaluation of the method by comparison with perfusion PET. J Nucl Med. 2005;46:736-744.

49. Nye JA, Hamill J, Tudorascu D, Carew J, Esteves F, Votaw JR. Comparison of low-pitch and respiratory-averaged CT protocols for attenuation correction of cardiac PET studies. Med Phys. 2009;36:1618-1623.

50. Martinez-Möller A, Zikic D, Botnar RM, et al. Dual cardiac-respiratory gated PET: implementation and results from a feasibility study. Eur J Nucl Med Mol Imaging. 2007;34:1447-1454.

51. Nye JA, Esteves F, Votaw JR. Minimizing artifacts resulting from respiratory and cardiac motion by optimization of the transmission scan in cardiac PET/CT. Med Phys. 2007;34:1901-1906.

52. Teräs M, Kokki T, Durand-Schaefer N, et al. Dual-gated cardiac PET-clinical feasibility study. Eur J Nucl Med Mol Imaging. 2010;37:505-516.

53. Bai C, Conwell R, Kindem J, et al. Phantom evaluation of a cardiac SPECT/ VCT system that uses a common set of solid-state detectors for both emission and transmission scans. J Nucl Cardiol. 2010;17:459-469.

54. Einstein AJ, Johnson LL, Bokhari S, et al. Agreement of visual estimation of coronary artery calcium from low-dose CT attenuation correction scans in hybrid PET/CT and SPECT/CT with standard Agatston score. J Am Coll Cardiol. 2010;56:1914-1921.

55. Schepis T, Gaemperli O, Koepfli P, et al. Use of coronary calcium score scans from stand-alone multislice computed tomography for attenuation correction of myocardial perfusion SPECT. Eur J Nucl Med Mol Imaging. 2007;34: 11-19.

56. Burkhard N, Herzog BA, Husmann L, et al. Coronary calcium score scans for attenuation correction of quantitative PET/CT ${ }^{13} \mathrm{~N}$-ammonia myocardial perfusion imaging. Eur J Nucl Med Mol Imaging. 2010;37:517-521.

57. Herzog BA, Husmann L, Landmesser U, Kaufmann PA. Low-dose computed tomography coronary angiography and myocardial perfusion imaging: cardiac hybrid imaging below 3mSv. Eur Heart J. 2009;30:644.

58. Nakazato R, Dey D, Gutstein A, et al. Coronary artery calcium scoring using a reduced tube voltage and radiation dose protocol with dual-source computed tomography. J Cardiovasc Comput Tomogr. 2009;3:394-400.

59. Scheffel H, Alkadhi H, Leschka S, et al. Low-dose CT coronary angiography in the step-and-shoot mode: diagnostic performance. Heart. 2008;94:1132-1137.

60. Weigold WG, Abbara S, Achenbach S, et al. Standardized medical terminology for cardiac computed tomography: a report of the Society of Cardiovascular Computed Tomography. J Cardiovasc Comput Tomogr. 2011;5:136-144.

61. Husmann L, Valenta I, Gaemperli O, et al. Feasibility of low-dose coronary CT angiography: first experience with prospective ECG-gating. Eur Heart J. 2008;29:191-197.

62. Javadi M, Mahesh M, McBride G, et al. Lowering radiation dose for integrated assessment of coronary morphology and physiology: first experience with stepand-shoot CT angiography in a rubidium 82 PET-CT protocol. $J$ Nucl Cardiol. 2008; 15:783-790.

63. Husmann L, Herzog BA, Gaemperli O, et al. Diagnostic accuracy of computed tomography coronary angiography and evaluation of stress-only single-photon emission computed tomography/computed tomography hybrid imaging: comparison of prospective electrocardiogram-triggering vs. retrospective gating. Eur Heart J. 2009;30:600-607.

64. Labounty TM, Leipsic J, Min JK, et al. Effect of padding duration on radiation dose and image interpretation in prospectively ECG-triggered coronary CT angiography. AJR. 2010;194:933-937.

65. Achenbach S, Marwan M, Ropers D, et al. Coronary computed tomography angiography with a consistent dose below $1 \mathrm{mSv}$ using prospectively electrocardiogram-triggered high-pitch spiral acquisition. Eur Heart J. 2010;31:340-346.

66. Leipsic J, Labounty TM, Heilbron B, et al. Estimated radiation dose reduction using adaptive statistical iterative reconstruction in coronary CT angiography: the ERASIR study. AJR. 2010;195:655-660.

67. Leipsic J, Labounty TM, Heilbron B, et al. Adaptive statistical iterative reconstruction: assessment of image noise and image quality in coronary CT angiography. AJR. 2010;195:649-654. 
68. He ZX, Hedrick TD, Pratt CM, et al. Severity of coronary artery calcification by electron beam computed tomography predicts silent myocardial ischemia. Circulation. 2000;101:244-251.

69. Moser KW, O'Keefe JH Jr. Bateman TM, McGhie IA. Coronary calcium screening in asymptomatic patients as a guide to risk factor modification and stress myocardial perfusion imaging. J Nucl Cardiol. 2003;10:590-598.

70. Berman DS, Wong ND, Gransar H, et al. Relationship between stress-induced myocardial ischemia and atherosclerosis measured by coronary calcium tomography. J Am Coll Cardiol. 2004;44:923-930.

71. Anand DV, Lim E, Raval U, Lipkin D, Lahiri A. Prevalence of silent myocardial ischemia in asymptomatic individuals with subclinical atherosclerosis detected by electron beam tomography. J Nucl Cardiol. 2004;11:450-457.

72. Raggi P, Berman DS. Computed tomography coronary calcium screening and myocardial perfusion imaging. J Nucl Cardiol. 2005;12:96-103.

73. Thompson RC, McGhie AI, Moser KW, et al. Clinical utility of coronary calcium scoring after nonischemic myocardial perfusion imaging. $\mathrm{J} \mathrm{Nucl} \mathrm{Car-}$ diol. 2005; 12:392-400.

74. Anand DV, Lim E, Hopkins D, et al. Risk stratification in uncomplicated type 2 diabetes: prospective evaluation of the combined use of coronary artery calcium imaging and selective myocardial perfusion scintigraphy. Eur Heart J. 2006;27:713-721.

75. Rosman J, Shapiro M, Pandey A, VanTosh A, Bergmann SR. Lack of correlation between coronary artery calcium and myocardial perfusion imaging. $\mathrm{J} \mathrm{Nucl}$ Cardiol. 2006;13:333-337.

76. Schuijf JD, Wijns W, Jukema JW, et al. A comparative regional analysis of coronary atherosclerosis and calcium score on multislice CT versus myocardial perfusion on SPECT. J Nucl Med. 2006;47:1749-1755.

77. Schepis T, Gaemperli O, Koepfli P, et al. Added value of coronary artery calcium score as an adjunct to gated SPECT for the evaluation of coronary artery disease in an intermediate-risk population. J Nucl Med. 2007;48:14241430.

78. Rozanski A, Gransar H, Wong ND, et al. Use of coronary calcium scanning for predicting inducible myocardial ischemia: influence of patients' clinical presentation. J Nucl Cardiol. 2007;14:669-679.

79. Rozanski A, Gransar H, Wong ND, et al. Clinical outcomes after both coronary calcium scanning and exercise myocardial perfusion scintigraphy. J Am Coll Cardiol. 2007;49:1352-1361.

80. Scholte AJ, Schuijf JD, Kharagjitsingh AV, et al. Different manifestations of coronary artery disease by stress SPECT myocardial perfusion imaging, coronary calcium scoring, and multislice CT coronary angiography in asymptomatic patients with type 2 diabetes mellitus. J Nucl Cardiol. 2008;15:503-509.

81. Chang SM, Nabi F, Xu J, et al. The coronary artery calcium score and stress myocardial perfusion imaging provide independent and complementary prediction of cardiac risk. J Am Coll Cardiol. 2009;54:1872-1882.

82. Blankstein R, Dorbala S. Adding calcium scoring to myocardial perfusion imaging: does it alter physicians' therapeutic decision making? J Nucl Cardiol. 2010;17:168-171.

83. Nabi F, Chang SM, Pratt CM, et al. Coronary artery calcium scoring in the emergency department: identifying which patients with chest pain can be safely discharged home. Ann Emerg Med. 2010;56:220-229.

84. Hacker M, Jakobs T, Matthiesen F, et al. Comparison of spiral multidetector CT angiography and myocardial perfusion imaging in the noninvasive detection of functionally relevant coronary artery lesions: first clinical experiences. $\mathrm{J} \mathrm{Nucl}$ Med. 2005;46:1294-1300.

85. Di Carli MF, Dorbala S, Curillova Z, et al. Relationship between CT coronary angiography and stress perfusion imaging in patients with suspected ischemic heart disease assessed by integrated PET-CT imaging. J Nucl Cardiol. 2007;14:799-809.

86. Gaemperli O, Schepis T, Kalff V, et al. Validation of a new cardiac image fusion software for three-dimensional integration of myocardial perfusion SPECT and stand-alone 64-slice CT angiography. Eur J Nucl Med Mol Imaging. 2007;34: 1097-1106.

87. Rispler S, Keidar Z, Ghersin E, et al. Integrated single-photon emission computed tomography and computed tomography coronary angiography for the assessment of hemodynamically significant coronary artery lesions. J Am Coll Cardiol. 2007;49:1059-1067.

88. Gaemperli O, Schepis T, Koepfli P, et al. Accuracy of 64-slice CT angiography for the detection of functionally relevant coronary stenoses as assessed with myocardial perfusion SPECT. Eur J Nucl Med Mol Imaging. 2007;34:11621171 .

89. Gallagher MJ, Ross MA, Raff GL, Goldstein JA, O'Neill WW, O'Neil B. The diagnostic accuracy of 64-slice computed tomography coronary angiography compared with stress nuclear imaging in emergency department low-risk chest pain patients. Ann Emerg Med. 2007;49:125-136.
90. Gaemperli O, Schepis T, Valenta I, et al. Functionally relevant coronary artery disease: comparison of 64-section CT angiography with myocardial perfusion SPECT. Radiology. 2008;248:414-423.

91. Sato A, Hiroe M, Tamura M, et al. Quantitative measures of coronary stenosis severity by 64-slice CT angiography and relation to physiologic significance of perfusion in nonobese patients: comparison with stress myocardial perfusion imaging. J Nucl Med. 2008;49:564-572.

92. Husmann L, Wiegand M, Valenta I, et al. Diagnostic accuracy of myocardial perfusion imaging with single photon emission computed tomography and positron emission tomography: a comparison with coronary angiography. Int J Cardiovasc Imaging. 2008;24:511-518.

93. Gaemperli O, Husmann L, Schepis T, et al. Coronary CT angiography and myocardial perfusion imaging to detect flow-limiting stenoses: a potential gatekeeper for coronary revascularization? Eur Heart J. 2009;30:2921-2929.

94. Haramati LB, Levsky JM, Jain VR, et al. CT angiography for evaluation of coronary artery disease in inner-city outpatients: an initial prospective comparison with stress myocardial perfusion imaging. Int J Cardiovasc Imaging. 2009;25:303-313

95. Santana CA, Garcia EV, Faber TL, et al. Diagnostic performance of fusion of myocardial perfusion imaging (MPI) and computed tomography coronary angiography. J Nucl Cardiol. 2009;16:201-211.

96. van Werkhoven JM, Schuijf JD, Gaemperli O, et al. Prognostic value of multislice computed tomography and gated single-photon emission computed tomography in patients with suspected coronary artery disease. J Am Coll Cardiol. 2009;53:623-632.

97. Pazhenkottil AP, Herzog BA, Husmann L, et al. Non-invasive assessment of coronary artery disease with CT coronary angiography and SPECT: a novel dose-saving fast-track algorithm. Eur J Nucl Med Mol Imaging. 2010;37:522527.

98. Ramakrishna G, Miller TD, Breen JF, Araoz PA, Hodge DO, Gibbons RJ. Relationship and prognostic value of coronary artery calcification by electron beam computed tomography to stress-induced ischemia by single photon emission computed tomography. Am Heart J. 2007;153:807-814.

99. Schenker MP, Dorbala S, Hong EC, et al. Interrelation of coronary calcification, myocardial ischemia, and outcomes in patients with intermediate likelihood of coronary artery disease: a combined positron emission tomography/computed tomography study. Circulation. 2008;117:1693-1700.

100. Tanenbaum SR, Kondos GT, Veselik KE, Prendergast MR, Brundage BH, Chomka EV. Detection of calcific deposits in coronary arteries by ultrafast computed tomography and correlation with angiography. Am J Cardiol. 1989;63:870-872.

101. Wexler L, Brundage B, Crouse J, et al. Coronary artery calcification: pathophysiology, epidemiology, imaging methods, and clinical implications-a statement for health professionals from the American Heart Association Writing Group. Circulation. 1996;94:1175-1192.

102. Sato A, Nozato T, Hikita $\mathrm{H}$, et al. Incremental value of combining 64-slice computed tomography angiography with stress nuclear myocardial perfusion imaging to improve noninvasive detection of coronary artery disease. $\mathrm{J} \mathrm{Nucl}$ Cardiol. 2010;17:19-26.

103. Min JK, Hachamovitch R, Rozanski A, Shaw LJ, Berman DS, Gibbons R. Clinical benefits of noninvasive testing: coronary computed tomography angiography as a test case. JACC Cardiovasc Imaging. 2010;3:305-315.

104. Hoffmann MH, Shi H, Schmitz BL, et al. Noninvasive coronary angiography with multislice computed tomography. JAMA. 2005;293:2471-2478.

105. Hachamovitch R, Hayes SW, Friedman JD, Cohen I, Berman DS. Comparison of the short-term survival benefit associated with revascularization compared with medical therapy in patients with no prior coronary artery disease undergoing stress myocardial perfusion single photon emission computed tomography. Circulation. 2003;107:2900-2907.

106. Shaw LJ, Berman DS, Maron DJ, et al. Optimal medical therapy with or without percutaneous coronary intervention to reduce ischemic burden: results from the Clinical Outcomes Utilizing Revascularization and Aggressive Drug Evaluation (COURAGE) trial nuclear substudy. Circulation. 2008;117:1283-1291.

107. Dorbala S, Hachamovitch R, Di Carli MF. Myocardial perfusion imaging and multidetector computed tomographic coronary angiography: appropriate for all patients with suspected coronary artery disease? J Am Coll Cardiol. 2006;48: 2515-2517.

108. Knuuti J. Cardiac hybrid imaging with low radiation dose. J Nucl Cardiol. 2008;15:743-744.

109. Kaufmann PA, Di Carli MF. Hybrid SPECT/CT and PET/CT imaging: the next step in noninvasive cardiac imaging. Semin Nucl Med. 2009;39:341-347.

110. Raff GL, Abidov A, Achenbach S, et al. SCCT guidelines for the interpretation and reporting of coronary computed tomographic angiography. J Cardiovasc Comput Tomogr. 2009;3:122-136. 
111. Killip T, Passamani E, Davis K. Coronary artery surgery study (CASS): a randomized trial of coronary bypass surgery-eight years follow-up and survival in patients with reduced ejection fraction. Circulation. 1985;72: V102-V109.

112. Sharir T, Germano G, Kang X, et al. Prediction of myocardial infarction versus cardiac death by gated myocardial perfusion SPECT: risk stratification by the amount of stress-induced ischemia and the poststress ejection fraction. $\mathrm{J} \mathrm{Nucl}$ Med. 2001;42:831-837.

113. Dorbala S, Vangala D, Sampson U, Limaye A, Kwong R, Di Carli MF. Value of vasodilator left ventricular ejection fraction reserve in evaluating the magnitude of myocardium at risk and the extent of angiographic coronary artery disease: a ${ }^{82} \mathrm{Rb}$ PET/CT study. J Nucl Med. 2007;48:349-358.

114. Dorbala S, Hachamovitch R, Curillova Z, et al. Incremental prognostic value of gated $\mathrm{Rb}-82$ positron emission tomography myocardial perfusion imaging over clinical variables and rest LVEF. JACC Cardiovasc Imaging. 2009;2: 846-854.

115. Hachamovitch R, Johnson JR, Hlatky MA, et al. The study of myocardial perfusion and coronary anatomy imaging roles in CAD (SPARC): design, rationale, and baseline patient characteristics of a prospective, multicenter observational registry comparing PET, SPECT, and CTA for resource utilization and clinical outcomes. J Nucl Cardiol. 2009;16:935-948.

116. Dey D, Slomka PJ, Hahn LJ, Kloiber R. Automatic three-dimensional multimodality registration using radionuclide transmission CT attenuation maps: a phantom study. J Nucl Med. 1999;40:448-455.

117. Slomka PJ. Software approach to merging molecular with anatomic information. J Nucl Med. 2004;45(suppl 1):36S-45S.

118. Slomka PJ, Cheng VY, Dey D, et al. Quantitative analysis of myocardial perfusion SPECT anatomically guided by coregistered 64-slice coronary CT angiography. J Nucl Med. 2009;50:1621-1630.

119. Gaemperli O, Schepis T, Valenta I, et al. Cardiac image fusion from stand-alone SPECT and CT: clinical experience. J Nucl Med. 2007;48:696-703.

120. Kajander S, Joutsiniemi E, Saraste M, et al. Cardiac positron emission tomography/computed tomography imaging accurately detects anatomically and functionally significant coronary artery disease. Circulation. 2010;122: 603-613.

121. Javadi MS, Lautamaki R, Merrill J, et al. Definition of vascular territories on myocardial perfusion images by integration with true coronary anatomy: a hybrid PET/CT analysis. J Nucl Med. 2010;51:198-203.

122. Wagner B, Anton M, Nekolla SG, et al. Noninvasive characterization of myocardial molecular interventions by integrated positron emission tomography and computed tomography. J Am Coll Cardiol. 2006;48:2107-2115.

123. Lautamäki R, Schuleri KH, Sasano T, et al. Integration of infarct size, tissue perfusion, and metabolism by hybrid cardiac positron emission tomography/ computed tomography: evaluation in a porcine model of myocardial infarction. Circ Cardiovasc Imaging. 2009;2:299-305.

124. Knuuti J. Integrated positron emission tomography/computed tomography (PET/CT) in coronary disease. Heart. 2009;95:1457-1463.

125. Tilkemeier PL, Cooke DC, Grossman GB, McCallister BD, Ward PR. American Society of Nuclear Cardiology Web site. Standardized reporting of radionuclide myocardial perfusion and function. Available at: http://www.asnc.org/ imageuploads/ImagingGuidelinesReportingJuly2009.pdf. Published 2009. Accessed March 1, 2012.

126. Douglas PS, Hendel RC, Cummings JE, et al. ACCF/ACR/AHA/ASE/ ASNC/HRS/NASCI/RSNA/SAIP/SCAI/SCCT/SCMR 2008 health policy statement on structured reporting in cardiovascular imaging. Endorsed by the Society of Nuclear Medicine and Molecular Imaging. Circulation. 2009; 119:187-200.

127. Hendel RC, Budoff MJ, Cardella JF, et al. ACC/AHA/ACR/ASE/ASNC/HRS/ NASCI/RSNA/SAIP/SCAI/SCCT/SCMR/SIR $2008 \mathrm{key}$ data elements and definitions for cardiac imaging: a report of the American College of Cardiology/ American Heart Association Task Force on Clinical Data Standards (Writing Committee to Develop Clinical Data Standards for Cardiac Imaging). J Am Coll Cardiol. 2009;53:91-124.

128. Bluemke DA, Achenbach S, Budoff M, et al. Noninvasive coronary artery imaging: magnetic resonance angiography and multidetector computed tomography angiography - a scientific statement from the American Heart Association Committee on Cardiovascular Imaging and Intervention of the Council on Cardiovascular Radiology and Intervention, and the Councils on Clinical Cardiology and Cardiovascular Disease in the Young. Circulation. 2008;118:586-606.

129. Husmann L, Tatsugami F, Aepli U, et al. Prevalence of noncardiac findings on low dose 64-slice computed tomography used for attenuation correction in myocardial perfusion imaging with SPECT. Int J Cardiovasc Imaging. 2009; 25:859-865.
130. Lehman SJ, Abbara S, Cury RC, et al. Significance of cardiac computed tomography incidental findings in acute chest pain. Am J Med. 2009;122:543-549.

131. ACR technical standard for diagnostic medical physics performance monitoring of computed tomography (CT) equipment. American College of Radiology Web site. Available at: http://www.acr.org/SecondaryMainMenuCategories/ quality_safety/guidelines/med_phys/ct_equipment.aspx. Published 2007. Accessed March 1, 2012.

132. Gould KL, Pan T, Loghin C, Johnson NP, Guha A, Sdringola S. Frequent diagnostic errors in cardiac PET/CT due to misregistration of CT attenuation and emission PET images: a definitive analysis of causes, consequences, and corrections. J Nucl Med. 2007;48:1112-1121.

133. Goetze S, Wahl RL. Prevalence of misregistration between SPECT and CT for attenuation-corrected myocardial perfusion SPECT. J Nucl Cardiol. 2007;14: 200-206.

134. Lautamäki R, Brown TL, Merrill J, Bengel FM. CT-based attenuation correction in ${ }^{82} \mathrm{Rb}$-myocardial perfusion PET-CT: incidence of misalignment and effect on regional tracer distribution. Eur J Nucl Med Mol Imaging. 2008;35:305-310.

135. Horii Y, Yoshimura N, Hori Y, et al. Relationship between heart rate and optimal reconstruction phase in dual-source CT coronary angiography. Acad Radiol. 2011;18:726-730.

136. Einstein AJ, Moser KW, Thompson RC, Cerqueira MD, Henzlova MJ. Radiation dose to patients from cardiac diagnostic imaging. Circulation. 2007;116: 1290-1305.

137. Kim KP, Einstein AJ, Berrington de Gonzalez A. Coronary artery calcification screening: estimated radiation dose and cancer risk. Arch Intern Med. 2009; 169:1188-1194.

138. Cerqueira MD, Allman KC, Ficaro EP, et al. Recommendations for reducing radiation exposure in myocardial perfusion imaging. J Nucl Cardiol. 2010;17:709-718.

139. Sharir T, Slomka PJ, Hayes SW, et al. Multicenter trial of high-speed versus conventional single-photon emission computed tomography imaging: quantitative results of myocardial perfusion and left ventricular function. $J$ Am Coll Cardiol. 2010;55:1965-1974.

140. DePuey EG, Bommireddipalli S, Clark J, Thompson L, Srour Y. Wide beam reconstruction "quarter-time" gated myocardial perfusion SPECT functional imaging: a comparison to "full-time" ordered subset expectation maximum. J Nucl Cardiol. 2009;16:736-752.

141. DePuey EG, Gadiraju R, Clark J, Thompson L, Anstett F, Shwartz SC. Ordered subset expectation maximization and wide beam reconstruction "half-time" gated myocardial perfusion SPECT functional imaging: a comparison to "full-time" filtered backprojection. J Nucl Cardiol. 2008;15:547-563.

142. Ali I, Ruddy TD, Almgrahi A, Anstett FG, Wells RG. Half-time SPECT myocardial perfusion imaging with attenuation correction. J Nucl Med. 2009;50:554-562.

143. Hausleiter J, Meyer T, Hadamitzky M, et al. Radiation dose estimates from cardiac multislice computed tomography in daily practice: impact of different scanning protocols on effective dose estimates. Circulation. 2006;113:1305-1310.

144. Hausleiter J, Meyer T, Hermann F, et al. Estimated radiation dose associated with cardiac CT angiography. JAMA. 2009;301:500-507.

145. Shuman WP, Branch KR, May JM, et al. Prospective versus retrospective ECG gating for 64-detector CT of the coronary arteries: comparison of image quality and patient radiation dose. Radiology. 2008;248:431-437.

146. International Commission on Radiological Protection. Radiation dose to patients from radiopharmaceuticals: Addendum 2 to ICRP publication 53. Ann ICRP. 1998;28:1-126.

147. International Commission on Radiological Protection. Radiation dose to patients from radiopharmaceuticals: a third addendum to ICRP publication 53, ICRP publication 106-approved by the Commission in October 2007. Ann ICRP. 2008;38:1-197.

148. Stabin MG. Proposed revision to the radiation dosimetry of ${ }^{82} \mathrm{Rb}$. Health Phys. 2010;99:811-813.

149. International Commission on Radiological Protection. ICRP publication 53: radiation dose to patients from radiopharmaceuticals. Ann ICRP. 1988;18:1-4.

150. Russell JR, Stabin MG, Sparks RB, Watson E. Radiation absorbed dose to the embryo/fetus from radiopharmaceuticals. Health Phys. 1997;73:756-769.

151. Stabin MG. Proposed addendum to previously published fetal dose estimate tables for ${ }^{18}$ F-FDG. J Nucl Med. 2004;45:634-635.

152. Maddahi J, Mendez R, Mahmarian JJ, et al. Prospective multicenter evaluation of rapid, gated SPECT myocardial perfusion upright imaging. J Nucl Cardiol. 2009;16:351-357.

\section{APPROVAL}

This practice guideline was approved by the Board of Directors of the SNMMI on November 15, 2012. 